\title{
GUC-Secure Join Operator in Distributed Relational Database
}

\author{
TIAN Yuan \\ Software School of Dalian University of Technology \\ tianyuan_ca!@sina.com
}

\begin{abstract}
Privacy-preserving SQL computation in distributed relational database is one of important applications of secure multiparty computation. In contrast with comparatively more works on privacy-preserving data-query in database, only few works deal with provably-secure privacy-preserving data manipulations, among which the join operator is the most powerful in generating new data (relation). We propose a very general cryptographic protocol framework for secure 2-party join computation based on anonymous IBE (identity-based encryption) scheme and its user private-keys blind generation techniques. This construction is provably GUC (generalized universally composable) secure in standard model with acceptable efficiency. In addition, an efficient instantiation based-on the anonymous Boyen-Waters IBE scheme is presented which user private-key's blind generation protocol may be of independent values.
\end{abstract}

Keywords: Secure Multiparty Computation; Distributed Relational Database; Join; Anonymous Identity-based Encryption; Generalized Universally Composable Security.

\section{INTRODUCTION}

Relational database (RDBMS) is one of the most widely deployed and mission-critical information systems. In addition, distributed relational database technology has become one of the major recent developments in this area [15]. In distributed RDBMS, unlike a single centralized RDBMS server, information is distributed among multiple sites and some pieces of this information are highly private for its owners. Therefore a natural secure multiparty computation problem arises in this setting: how to generate the useful information while keeping all participiating sites' data privacy?

In RDBMS information is modeled as relations or tables. Such highly structured information is processed by the powerful relational algebra operators, e.g., (constrained) selection, projection, product and join operators, which are applied to one or multiple tables to generate new table [7]. Set operators, e.g., union, intersection, insert, etc., are also regarded as extended relational algebra operators. All these have been formally standardized as the RDBMS programming language SQL( Structured Query Language) which is widely used even in distributed RDBMS systems.

Motivations So far lots of works have been done to develop solutions to secure SQL computations in distributed RDBMS and the most majority comes from database rather than cryptography community. However, from the cryptography theorist's perspective, only few of them are reliable: most works, although highly heuristic, do not have precise security analysis or even without precise security definitions. As a (unfortunate) result, most of them are actually either insecure under the standard security definition currently accepted by cryptographists or unable-to-be-proved. In particular, since the SQL-operator is always called by high-level programs, its security must be preserved no matter how it is called and combined with any other (maybe malicious) running contexts. In other words, secure solutions to SQL-operators must be provably UC or GUC secure [4-5]. But with our knowledge 
none of such solution exists in this area. For this reason, we won't further review these works from database community (interested readers can refer the survey [9] and its references) and only focus on provably secure solutions.

Among the provably secure solutions to distributed data processing (not limited to distributed RDBMS), most of them are focused on data query(i.e., read-only) rather than data manipulation operators. Although data query is one of the most important and frequently-used operations in distributed systems, lack of secure solutions to data manipulation operators will become a bottleneck in deploying highly security-critical applications. Roughly speaking, secure data query solutions are applied on encrypted data (e.g., $[1,2,11,16-17]$, many approaches are not limited to relational data model) to extract the desired information via smartly designed trapdoor machenisms. All these solutions can provide techniques for constructing secure SQL-query operators such as the most frequently-used constrained selection operators. Generally speaking, secure data manipulation is more technically involved than data query schemes and so far there are indeed few works on this problem, among which $[10,12-13]$ provide the provably secure solutions. $[10,13]$ can be also regarded as the secure solution to SQL set operators, but none of them reaches Canetti's UC/GUC-security [4-5] ([10] works in two relaxed adversary models to achieve security of "half-simulatability" and "full-simulatability against covert adversaries"; [13] is provably secure in terms of full-simulatability which is still strictly weaker than UC/GUC-security). With our knowledge, there're no satisfactory secure solutions to important relational data manipulation operators such as join and its variants.

In this paper we focus on secure join computation. Join is a powerful SQL operator to generate a new table from existing tables in RDBMS. For example, given two tables, Incom with attributes "customer_id"and "income", Debt with attributes "customer_id" and "debt", then the joint operator Join(customer_id: Incom,Debt) produces a new table with attributes "customer_id", "income" and "debt" which entries are derived from the product of the entries in table Incom and Debt which have the same values in customer_id field. For instance, suppose Incom has entries $\{(\mathrm{c} 1,2500),(\mathrm{c} 2,3000),(\mathrm{c5}), 1010),(\mathrm{c} 6,2000)\}$, Debt has entries $\{(\mathrm{c} 2,19000),(\mathrm{c} 4,7000),(\mathrm{c5}), 88),(\mathrm{c} 7,100)\}$, then the above join operator outputs the entries $\{(\mathrm{c} 2,3000,19000),(\mathrm{c} 5,1010,88)\}$. Join computation with equality constraints on multiple common attributes is also useful in practice.

Furthermore, if the table Incom and Debt are stored at different sites and the information about those uncommon customers must be kept secret to each other site, this join operator must be securely implemented in consistency with the cryptographic multiparty computation definition, i.e., each site knows nothing about the other table beyond the join operator's output.

Contributions In this paper we construct a GUC-secure [4-5] protocol for join computation in standard model with acceptable efficiency. Like many theoretical works, we focus on the 2-party case. Our approach is very general based-on the anonymous IBE scheme and it's user private-key's blind generation techniques (i.e., to generate the correct user private-key usk $(a)=\mathrm{UKG}(m s k, a)$ for a user without leaking the user-id $a$ to the key-generator). The protocol's high-level description is simple: let $\Pi=\left(\right.$ Setup,UKG,E,D) be an IBE scheme both anonymous and data-private, $M_{0}$ be some publicly-known plaintext. Site $\mathrm{P}_{1}$ owns the table $\mathrm{X}_{1}$ with attributes $w$ and $x$ and site $\mathrm{P}_{2}$ owns table $\mathrm{X}_{2}$ 
with attributes $w$ and $y$. The goal is securely computing $\operatorname{Join}\left(w: \mathrm{X}_{1}, \mathrm{X}_{2}\right)$ and outputing at $\mathrm{P}_{2}{ }^{1}$. suppose $\mathrm{X}_{1}$ is filled with entries $\left\{\left(w_{1}, x_{1}\right),\left(w_{2}, x_{2}\right),\left(w_{3}, x_{3}\right),\left(w_{4}, x_{4}\right)\right\}$ and $\mathrm{X}_{2}$ is filled with $\left\{\left(w_{2}, y_{2}\right),\left(w_{4}, y_{4}\right)\right.$, $\left.\left(w_{5}, y_{5}\right),\left(w_{7}, y_{7}\right)\right\}$ where $w_{i}$ 's, $x_{i}$ 's and $y_{i}$ 's are values of the attribute $w, x$ and $y$ respectively. $\mathrm{P}_{1}$ generates IBE's master public/secret-key (mpk,msk), sends $m p k$ and all $\xi_{i}=\mathrm{E}\left(m p k, w_{i}, x_{i} \| M_{0}\right)(i=1,2,3,4)$ to $\mathrm{P}_{2}$. When $\mathrm{P}_{2}$ tries to decipher each $\xi_{i}$ by private-keys $u s k\left(w_{2}\right)$, usk $\left(w_{4}\right), u s k\left(w_{5}\right)$ and $u s k\left(w_{7}\right)$ (obtained via $\Pi$ 's user private-keys blind generation protocol), only $u s k\left(w_{2}\right)$ and $u s k\left(w_{4}\right)$ can succeed in obtaining the plaintext with a suffix $M_{0}$. As a result, $\mathrm{P}_{2}$ gets $\mathrm{X}_{1}$ 's entries $\left\{\left(w_{2}, x_{2}\right),\left(w_{4}, x_{4}\right)\right\}$ and can now get the result of $\operatorname{Join}\left(w: \mathrm{X}_{1}, \mathrm{X}_{2}\right)$, i.e., $\left\{\left(w_{2}, x_{2}, y_{2}\right),\left(w_{4}, x_{4}, y_{4}\right)\right\}$, by a local join computation. Note that $\Pi$ 's anonymity and data-privacy prevents $\mathrm{P}_{2}$ from knowing anything about $\mathrm{X}_{1}$ beyond $\left\{\left(w_{2}, x_{2}\right),\left(w_{4}, x_{4}\right)\right\}$ while the private-key generation protocol's blindness prevents $\mathrm{P}_{1}$ from knowing anything about $\mathrm{X}_{2}$.

This protocol's incorrectness probability is not 0 , but by chosing $M_{0}$ lengthy enough, e.g., 128-bit, its incorrectness probability can be negligible in practice.

To be GUC-secure, the formal constrution is more involved (section 3). It is constant-round in communications and linear-size in message-complexity. In computation-complexity, one party is $\mathrm{O}\left(\mathrm{N}_{1}+\mathrm{N}_{2}\right)$ and the other is $\mathrm{O}\left(\mathrm{N}_{1} \mathrm{~N}_{2}\right)$ encryptions/decriptions where $\mathrm{N}_{1}, \mathrm{~N}_{2}$ are each party's private table's cardinality. Note that $\mathrm{O}\left(\mathrm{N}_{1} \mathrm{~N}_{2}\right)$ is also local join operator's computation complexity [7], i.e., neglecting a constant factor our construction's efficiency is asymptotically the same as that of conventional join operator.

The formal construction is also well-modularized, only executing few zero-knowledge proofs of knowledge which can be efficiently instantiated. Most importantly and distinctively, our construction is provably GUC-secure against malicious adversaries assuming static corruptions in the ACRS(augmented common reference string) model [5]. For this goal we introduce a notion of identity-augmented non-malleable zero-knowledge proofs of knowledge which may be of independent values. This construction can be also enhanced to be GUC-secure against malicious adversaries assuming adaptive corruptions in erasure model by some slight modifications.

Why IBE? Although there can be other approaches to the same solution, our approach has the good potential to deal with more complicated cases within a unified framework. In fact what we deal with in this paper is just the most simple (also most frequently-used) case: equijoin. A general join operator (called theta-join) involves some condition on attributes from each of its argument tables, i.e., $\operatorname{Join}\left(\theta(\boldsymbol{a}, \boldsymbol{b}): \mathrm{X}_{1}, \mathrm{X}_{2}\right)$ where $\theta$ is a predicate, $\boldsymbol{a}, \boldsymbol{b}$ are vectors of attributes of $\mathrm{X}_{1}$ and $\mathrm{X}_{2}$ respectively, e.g., $\boldsymbol{a}=\left(a^{(1)}, a^{(2)}\right), \boldsymbol{b}=\left(b^{(1)}, b^{(2)}\right)$, for equijoin $\theta(\boldsymbol{a}, \boldsymbol{b}) \equiv a^{(1)}=b^{(1)} \wedge a^{(2)}=b^{(2)}$ but in general $\theta(\boldsymbol{a}, \boldsymbol{b})$ can be any predicate, e.g., $a^{(1)}<b^{(1)}, a^{(1)}+a^{(2)} \geq b^{(1)}+b^{(2)}, a^{(1)}<b^{(1)} \wedge a^{(2)}=b^{(2)}$, etc. We believe that some further extensions to recently proposed ABE schemes(e.g., $[11,17])$ which are powerful generalizations of IBE will be helpful to solve such secure SQL computation problems while keeping the general protocol framework in section 3 unchanged, which has obvious advantages in practice.

Organization Section 2 briefly presents all required important notions and facts, including an enhanced concept of zero-knowledge proofs of knowledge (def. 2.4). Section 3 presents the general construction and section 4 instantiates it via the anonymous Boyen-Waters IBE scheme. For space limitations, detailed proofs are presented in Appdenix B and C. Appendix D presents a general and

\footnotetext{
${ }^{1}$ In most cases a distributed SQL-transaction outputs its final result at some particular site.
} 
efficient construction of the required zero-knowledge proof protocol.

\section{NOTATIONS, DEFINITIONS AND TOOLS}

P.P.T. means "probabilistic polynomial-time", $x \| \mathrm{y}$ means string $x$ and $y$ in concatenation, $|x|$ means string $x$ 's size(in bits) and $|\mathrm{X}|\left(\mathrm{X}\right.$ is a set) means $\mathrm{X}$ 's cardinality, $x \leftarrow{ }^{\$} \mathrm{X}$ means randomly selecting $x$ from the domain $\mathrm{X} . k$ denotes the complexity parameter. $\approx^{\mathrm{PPT}}$ stands for computational indistinguishability and $\approx$ for perfect indistinguishability.

\subsection{Secure Join Computation and Its GUC Security}

Briefly speaking, GUC-security means that any adversary attacking the real-world protocol can be efficiently simulated by an adversary attacking the ideal-world functionality, both have the outputs indistinguishable by the (malicious) environment. For space limitations, we assume the reader's familiarity with the whole theory in [4-6] and only provide necessary descriptions with respect to the secure join computation problem here.

Similar to many theoretical works, we focus on the 2-party scenario. Furthermore, in this paper we only consider the horizontal distribution setting in which each table is at a site as a whole, none table is separated among different sites. Let $\mathrm{X}_{1}$ and $\mathrm{X}_{2}$ denote the tables with attributes $w, u^{(1)}$ and $w$, $u^{(2)}$ respectively, $w$ is the common attribute and for equijoin we consider only single such common attribute without loss of generality (in case of multiple common attributes, e.g., $v$ and $w$, we simply consider a imaginary single attribute $v \| w$ which values are just the concatenation of values of $v$ and $w$ ). The ideal cryptographic functionality to perform equijoin computation on $X_{1}$ and $X_{2}$ with equality constraint on $w$ is defined as

$$
F_{\text {Join }}:\left(\mathrm{X}_{1}, \mathrm{X}_{2}\right) \rightarrow\left(|w|_{2},\left|\mathrm{X}_{1}\right||| \operatorname{Join}\left(w: \mathrm{X}_{1}, \mathrm{X}_{2}\right)\right)
$$

where $|w|_{2}$ means the number of different values of $w$ in $\mathrm{X}_{2}$ and $\operatorname{Join}\left(w: \mathrm{X}_{1}, \mathrm{X}_{2}\right)$ means the reault of ideal relational join computation. More precisely, let $\mathrm{P}_{1} *, \mathrm{P}_{2} *$ be parties in ideal model with private tables $\mathrm{X}_{1}$ and $\mathrm{X}_{2}$ respectively, $\mathrm{N}_{1}=\left|\mathrm{X}_{1}\right|, \mathrm{N}_{2}=\left|\mathrm{X}_{2}\right|, S$ be the adversary in ideal model, the ideal model works as follows:

On receiving message (sid,"input", $\left.\mathrm{P}_{1}{ }^{*}, \mathrm{X}_{1}\right)$ from $\mathrm{P}_{1}{ }^{*}, F_{\text {Join }}$ records $\mathrm{X}_{1}$ and sends message (sid,"input", $\mathrm{N}_{1}$ ) to $\mathrm{P}_{2} *$ and $\mathrm{S}$; On receiving message (sid, "input", $\mathrm{P}_{2}{ }^{*}, \mathrm{X}_{2}$ ) from $\mathrm{P}_{2}{ }^{*}, F_{\text {Join }}$ records $\mathrm{X}_{2}$ and sends (sid, “input", $\left.|w|_{2}\right)$ to $\mathrm{P}_{1} *$ and $S$.

On receiving message (sid,“equijoin”, $\left.\mathrm{P}_{2}{ }^{*}\right)$ from $\mathrm{P}_{2}, F_{\text {Join }}$ responses $\mathrm{P}_{2}{ }^{*}$ with message (sid,"equijoin", $\operatorname{Join}\left(w: \mathrm{X}_{1}, \mathrm{X}_{2}\right)$ ).

At last $\mathrm{P}_{1} *$ outputs $|w|_{2}, \mathrm{P}_{2} *$ outputs $\mathrm{N}_{1}|| \operatorname{Join}\left(w: \mathrm{X}_{1}, \mathrm{X}_{2}\right)$.

Let $\psi$ be the real-world protocol, each party $\mathrm{P}_{i}$ of $\psi$ corresponds to an ideal-world party $\mathrm{P}_{i}{ }^{*} . A$ is the real-world adversary attacking $\psi, Z$ is the environment in which the real protocol/ideal functionality executes. According to [4-5], $Z$ is a P.P.T. machine modeling all malicious behaviors against the protocol's execution. $Z$ is empowered to provide inputs to parties and interacts with $A$ and $S$, e.g., $Z$ gives special inputs or instructions to $A / S$, collects outputs from $A / S$ to make some analysis, etc. In UC theory [4], $Z$ cannot access parties' shared functionality (such shared functionality is specified in specific protocol) while in the improved GUC theory [5] $Z$ is enhanced to do this, i.e., to 
provide inputs to and get outputs from the shared functionality. As a result, in GUC theory $Z$ is strictly stronger and more realistic than in UC theory.

Let output $(\psi, A)$ denote the outputs (as a joint stochastic variable)from $\psi$ 's parties $\mathrm{P}_{1}, \mathrm{P}_{2}$ under $\mathrm{Z}$ and $A$, output $\left(F_{\text {Join }}, S\right)$ denote the similar thing under $Z$ and $S$. During the real/ideal protocol's execution, $Z$ (as an active distinguisher) interacts with $A / S$ and raises its final output, w.l.o.g., 0 or 1. Such output is denoted as $Z\left(\operatorname{output}_{\mathrm{Z}}(\psi, A), u\right)$ and $Z\left(\operatorname{output}_{\mathrm{Z}}\left(F_{\text {Join }}, S\right), u\right)$ respectively, where $u$ is the auxiliary information.

Definition 2.1(GUC security [5]) If for any P.P.T. adversary $A$ in real-world, there exists a P.P.T. adversary $S$ (called $A$ 's simulator) in ideal-world, both corrupt the same set of parties, such that for any environment $Z$ the function $\left|\mathrm{P}\left[Z\left(\operatorname{output}_{\mathrm{Z}}(\psi, A), u\right)=1\right]-\mathrm{P}\left[Z\left(\operatorname{output}_{\mathrm{Z}}\left(F_{\text {Join }}, S\right), u\right)=1\right]\right|$ is negligible in complexity parameter $k$ (hereafter denote this fact as $\operatorname{output}_{\mathrm{Z}}(\psi, A) \approx^{\mathrm{PPT}} \operatorname{output}_{\mathrm{Z}}\left(F_{\text {Join }}, S\right)$ ), then we define that $\psi$ GUC-emulates $F_{\text {Join }}$ or say $\psi$ is GUC-secure, denoted as $\psi \rightarrow{ }^{\text {GUC }} F_{\text {Join }}$.

The most significant property of GUC-security is the universal composition theorem. Briefly speaking, given protocols $\varphi_{2}, \varphi_{1}$ and $\psi\left(\varphi_{1}\right)$ where $\psi\left(\varphi_{1}\right)$ is the so-called $\varphi_{1}$-hybrid protocol, if $\varphi_{2} \rightarrow{ }^{\mathrm{GUC}} \varphi_{1}$ then (under some technical conditions, e.g., subroutine-respecting) $\psi\left(\varphi_{2} / \varphi_{1}\right) \rightarrow{ }^{\mathrm{GUC}} \psi\left(\varphi_{1}\right)$ where $\psi\left(\varphi_{2} / \varphi_{1}\right)$ is a protocol in which every call to the subprotocol $\varphi_{1}$ is replaced with a call to $\varphi_{2}$. This guarantees that a GUC-secure protocol can be composed in any execution context while still preserving its proved security. A similar consequence is also ture in UC theory but with some serious constraints. All details are presented in [4-5]. ACRS model is defined in [5]'s sec.4 and repeated in Appendix A in our paper.

\subsection{IBE Scheme, Its Anonymity and Blind User-Private Key Generation Functionality}

In addition to data-privacy, anonymity(key-privacy) is another valuable property for public-key encryption schemes [1]. An IBE scheme $\Pi=($ Setup, UKG, E, D) is a group of P.P.T. algorithms, where Setup takes as input the complexity parameter $k$ to generate master public/secret-key pair ( $m p k, m s k$ ), UKG takes as input $m s k$ and user's id $a$ to generate $a$ 's user private-key $u s k(a)$; E takes $(m p k, a, M)$ as input where $M$ is the message plaintext to generate ciphertext $y$, D takes $(m p k, u s k(a), y)$ as input to do decryption. Altogether these algorithms satisfy the consistency property: for any $k, a$ and $M$

$\mathrm{P}[(m p k, m s k) \leftarrow \operatorname{Setup}(k) ; u s k(a) \leftarrow \mathrm{UKG}(m s k, a) ; y \leftarrow \mathrm{E}(m p k, a, M): \mathrm{D}(m p k, u s k(a), y)=M]=1$

Definition 2.2(IBE Scheme's Chosen Plaintext Anonymity [1]) Given an IBE scheme $\Pi=($ Setup, UKG,E,D), for any P.P.T. attacker $A=\left(A_{1}, A_{2}\right)$ consider the following experiment $\operatorname{Exp}_{\Pi, A}^{A N O \_C P A}(k)$ :

$$
\begin{aligned}
& (m p k, m s k) \leftarrow \operatorname{Setup}(k) ; \\
& \left(M^{*}, a_{0}^{*}, a_{1}{ }^{*}, S t\right) \leftarrow A_{1} \mathrm{UKG}(m s k, .)(m p k), a_{0}{ }^{*} \neq a_{1} * ; \\
& b \leftarrow{ }^{\$}\{0,1\} ; \\
& y^{*} \leftarrow \mathrm{E}\left(m p k, a_{b}{ }^{*}, M^{*}\right) ; \\
& d \leftarrow A_{2}{ }^{\mathrm{UKG}(m s k, .)}\left(S t, y^{*}\right) ; \\
& \operatorname{output}(d \oplus b) ;
\end{aligned}
$$

$A$ is contrained not to query its oracle $\operatorname{UKG}\left(m s k\right.$,.) with $a_{0} *$ and $a_{1} *$. Define $A d v_{\Pi, A}^{A N O_{-} C P A}$ as $\left|2 P\left[\operatorname{Exp}_{\Pi, A_{-}}^{A N O_{-} C P A}(k)=1\right]-1\right|$. If $A d v_{\Pi, A_{-}}^{A N O_{-} C P A}$ is negligible in $k$ for any P.P.T. $A$ then $\Pi$ is defined as 
anonymous against chosen plaintext attack or ANO_CPA for short. In the above, if $M^{*}, a_{0}^{*}, a_{1}^{*}$ are generated independent of $m p k$ then $\Pi$ is called id-selective ANO_CPA .

Denote $\max _{A \in P . P . T .} A d v_{\Pi, A}^{A N O_{-} C P A}(k)$ as $A d v_{\Pi}^{A N O_{-} C P A}(k)$ or $A d v_{\Pi}^{A N O_{-} C P A}(t, q)$ where $t$ is the adversary's maximum time-complexity and $q$ is the maximum number of queries for the UKG-oracle.

Now we present the ideal functionality $F_{\text {Blind-UKG }}^{\Pi}$ for an IBE scheme $\Pi$ 's user private-key blind generation(note: even IBE scheme is not anonymous such functionality still makes sense. However, in this paper only anonymous IBE's such protocol is needed). In the ideal model, one party generates(just

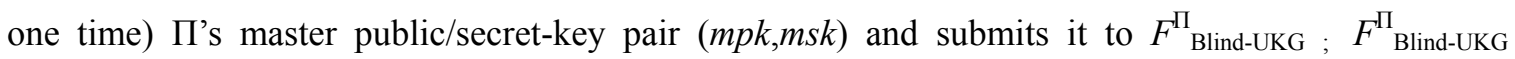
generates $u s k(a)=\mathrm{UKG}(m s k, a)$ for another party who submits its private input $a$ (this computation can take place any times and each time for a new $a$ ), revealing nothing about $a$ to the party who provides ( $m p k, m s k$ ) except how many private-keys are generated. Formally, let $S$ be the ideal adversary, $\mathrm{P}_{1}$ *, $\mathrm{P}_{2}{ }^{*}$ the ideal party, sid and ssid the session-id and subsession-id respectively, the ideal model works as follows:

$\mathrm{P}_{1} *$ selects randomness $\rho$ and computes (mpk,msk) $\leftarrow \operatorname{Setup}(\rho)$, sends the message (sid, $m p k\|m s k\| \rho)$ to $F_{\text {Blind-UKG }}^{\Pi} ; F_{\text {Blind-UKG }}^{\Pi}$ sends message (sid, mpk) to $\mathrm{P}_{2}{ }^{*}$ and $S$;

On receiving a message ( $\operatorname{sid}|| \mathrm{ssid}, a)$ from $\mathrm{P}_{2}{ }^{*}$ (ssid and a are fresh everytime), in

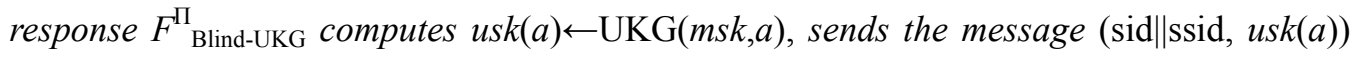
to $\mathrm{P}_{2}{ }^{*}$ and the message $(\operatorname{sid} \| \operatorname{ssid}, n)$ to $\mathrm{P}_{1} *$ and $S$, where $n$ is initialized to be 0 and increased by 1 everytime the computation takes place.

At last, $\mathrm{P}_{1}$ * outputs its last $n, \mathrm{P}_{2} *$ outputs all its obtained usk $(a)$ 's.

\section{3 (Identity-Augmented) Non-Malleable Zero-Knowledge Proofs of Knowledge}

This subsection presents the concept of zero-knowledge proofs of knowledge following $[8,14]$ with slight symbolic modifications. Let $L$ be a NP language, $R$ is its associated P-class binary relation. i.e., $x \in L$ iff there exists $w$ such that $R(x, w)=1$. Let $A, B$ be two machines, then $A(x ; B)_{[\sigma]}$ represents $A$ 's output due to its interactions with $B$ under a public common input $x$ and common reference string (c.r.s.) $\sigma, \operatorname{tr}_{\mathrm{A}, \mathrm{B}}(x)_{[\sigma]}$ represents the transcripts due to interactions between $A$ and $B$ under a common input $x$ and c.r.s. $\sigma$. When we emphasize $A$ 's private input, say $y$, we also use the expression $A_{y}(x ; B)_{[\sigma]}$ and $\operatorname{tr}_{\mathrm{A}(y), \mathrm{B}}(x)_{[\sigma]}$ respectively. Let $A=\left(A_{1}, A_{2}\right), B$ and $C$ be machines where $A_{1}$ can coordinate with $A_{2}$ by transferring status information to it, then $\left(\left\langle B, A_{1}\right\rangle,\left\langle A_{2}, C\right\rangle\right)$ represents the interaction between $A_{1}$ and $B$, (maybe concurrently) $A_{2}$ and $C$. Due to such interactions, let $t r$ be the transcripts between $A_{2}$ and $C, u$ be the final output from $A_{2}$ and $v$ be the final output form $C$, then $\left(<B, A_{1}>,<A_{2}, C>\right.$ )'s output is denoted as $(u, t r, v)$.

Two transcripts $t r_{1}$ and $t r_{2}$ are matched each other, if $t r_{1}$ and $t r_{2}$ are the same message sequence(consisted of the same messages in the same order) and the only difference is that any corresponding messages are in the opposite directions.

Let $A$ be a machine, the symbol $A$ represents such a machine which accepts two kinds of instructions: the first one is in the form of ("start", $i, x, w)$ and $A$ in response starts a new instance of $A$, associates it with a unique name $i$ and provides it with public input $x$ and private input $w$; the second is in form of ("message", $i, m)$ and $A$ in response sends message $m$ to instance $A_{i}$ and then returns $A_{i}$ 's 
response to $m$.

Definition 2.3(Zero-Knowldeg Proof and Non-Malleable Zero-Knowledge Proof Protocol [14]) $\mathrm{ZPoK}_{\mathrm{R}}=\left(\mathrm{D}_{c r s}, \mathrm{P}, \mathrm{V}, \mathrm{Sim}\right)$ where $\mathrm{Sim}=\left(\mathrm{Sim}_{1}, \mathrm{Sim}_{2}\right)$ is a group of P.P.T. algorithms, $k$ is complexity parameter, $\mathrm{D}_{c r s}$ takes $k$ as input and generates c.r.s. $\sigma$; $\mathrm{P}$ is called prover, takes $(\sigma, x, w)$ as input where $R(x, w)=1$ and generates a proof $\pi ; \mathrm{V}$ is called verifier, takes $(\sigma, x)$ as input and generates 0 or 1 ; $\operatorname{Sim}_{1}(k)$ generates $(\sigma, s), \operatorname{Sim}_{2}$ takes $x \in L$ and $(\sigma, s)$ as input and generates the simulation. All algorithms except $\mathrm{D}_{\text {crs }}$ and $\operatorname{Sim}_{1}$ take the c.r.s. $\sigma$ as one of their inputs, so $\sigma$ is no longer explicitly included in all the following expressions unless for emphasis. Now $Z \mathrm{ZPK}_{R}$ is defined as a zero-knowledge proof protocol for relation $R$, if the following properties are all satisfied:

(1) For any $x \in L$ and $\sigma \leftarrow \mathrm{D}_{c r s}$, it's always true that $\mathrm{P}\left[V(x ; P)_{[\sigma]}=1\right]=1$;

(2) For any P.P.T. algorithm $A, x \notin \mathrm{L}$ and $\sigma \leftarrow \mathrm{D}_{c r s}$, it's always true that $\mathrm{P}\left[V(x ; A)_{[\sigma]}=1\right]=0^{2}$;

(3) For any P.P.T. algorithm $A$ which outputs 0 or 1 , let $\varepsilon$ be empty string, the function

$$
\left|\mathrm{P}\left[\sigma \leftarrow \mathrm{D}_{c r s} ; b \leftarrow A(\varepsilon ; \mathrm{P})_{[\sigma]}: b=1\right]-\mathrm{P}\left[(\sigma, \mathrm{s}) \leftarrow \operatorname{Sim}_{1}(k) ; b \leftarrow A\left(\varepsilon ; \operatorname{Sim}_{2}(\mathrm{~s})\right)_{[\sigma]}: b=1\right]\right|
$$

is always negligible in $k$, where we emphasize the fact by $\operatorname{symbol}_{2} \operatorname{Sim}_{2}(s)$ that all $\operatorname{Sim}_{2}$ instances have the same $s$ as one of their inputs.

The non-malleable zero-knowledge proof protocol for relation $R$ is defined as $\mathrm{NMZPoK}_{\mathrm{R}}=$ $\left(\mathrm{D}_{c r s}, \mathrm{P}, \mathrm{V}, \mathrm{Sim}, \mathrm{Ext}\right)$ where $\mathrm{Sim}=\left(\operatorname{Sim}_{1}, \operatorname{Sim}_{2}\right), \mathrm{Ext}=\left(\mathrm{Ext}_{1}, \mathrm{Ext}_{2}\right)$ and $\left(\mathrm{D}_{c r s}, \mathrm{P}, \mathrm{V}, \mathrm{Sim}\right)$ is a zero-knowledge proof protocol for relation $R$ as above, P.P.T. algorithm $\operatorname{Ext}_{1}(k)$ generates $(\sigma, s, \tau)$ and the interactive P.P.T. machine Ext $_{2}$ (named as witness extractor) takes $(\sigma, \tau)$ and protocol's transcripts as its input and extracts $w$, and all the following properties hold:

(4) The distribution of the first output of $\operatorname{Sim}_{1}$ is identical to that of Ext

(5) For any $\tau$, the distribution of the output of $\mathrm{V}$ is identical to that of Ext 2 's restricted output which does not include the extracted value $(w)$;

(6) There exists a negligible function $\eta(k)$ (named as knowledge-error function) such that for any P.P.T. algorithm $A=\left(A_{1}, A_{2}\right)$ it's true that

$\mathrm{P}\left[(\sigma, s, \tau) \leftarrow \operatorname{Ext}_{1}(k) ; \quad(x, t r,(b, w)) \leftarrow\left(<\operatorname{Sim}_{2}(s), A_{1}>,<A_{2}, \operatorname{Ext}_{2}(\tau)>\right)_{[\sigma]}: \quad b=1 \wedge R(x, w)=1 \wedge t r\right.$ doesn't match any transcript generated by $\operatorname{Sim}_{2}(s)$ ]

$>\mathrm{P}\left[(\sigma, s) \leftarrow \operatorname{Sim}_{1}(k) ; \quad(x, t r, b) \leftarrow\left(<\operatorname{Sim}_{2}(\mathrm{~s}), A_{1}>,<A_{2}, \mathrm{~V}>\right)_{[\sigma]}: \quad b=1 \wedge t r\right.$ doesn't match any transcript generated by $\left.\operatorname{Sim}_{2}(\mathrm{~s})\right]$ - $\eta(k)$.

It's easy to see that $\mathrm{NMZPoK}_{\mathrm{R}}$ is a zero-knowledge proof of knowledge. [8,14] developed an efficient method to derive non-malleable zero-knowledge proof protocols based-on simulation-sound tag-based commitment schemes and the so-called $\Omega$-protocols (proposed in [14]). In order to achieve GUC-security in our construction, we need to further enhance NMZPoK to the concept of identity-augmented non-malleable zero-knowldege proof protocol(IA-NMZPoK) as follows.

Definition 2.4(IA-NMZPoK Protocol for Relation $R$ ) The IA-NMZPoK Protocol for relation $R$, IA-NMZPoK $R=(D, S e t u p, U K G, P, V, S i m, E x t)$ where $\operatorname{Sim}=\left(\operatorname{Sim}_{1}, \operatorname{Sim}_{2}\right)$ and $\operatorname{Ext}=\left(\operatorname{Ext}_{1}, \operatorname{Ext}_{2}\right)$, is a group of P.P.T. algorithms. Setup $(k)$ generates master public/secret-key pair (mpk,msk), UKG $(m s k, i d)$ generates $i d$ 's private-key usk(id) where $i d \in\{P, V\}$ (the prover's and verifier's identity). Sim 1 takes $u s k(V)$ as input, $\operatorname{Ext}_{1}$ takes $u s k(P)$ as input. All algorithms except Setup take $(m p k, \sigma)$ as one of its

\footnotetext{
${ }^{2}$ Strictly this protocol should be called "zero-knowledge argument", however, such difference is not essential in this paper so we harmlessly abuse the terminology.
} 
inputs(so it no longer explicitly appears). The protocol has the same properties as $R$ 's NMZPoK protocol in definition 2.3 .

Note that by this definition an IA-NMZPoK protocol works in ACRS model [5] which ACRS is its $m p k$. In addition, only the corrupt verifier can run $\operatorname{Sim}\left(\operatorname{Sim}_{1}\right.$ taking $u s k(V)$ as input) and only the corrupt prover can run Ext (Ext taking $u s k(P)$ as input). This is exactly what is required in ACRS model. Given a relation $R$, a general and efficient construction of IA-NMZPoK protocol for $R$ is presented in Appendix D.

\subsection{Commitment Scheme}

We need the non-interactive identity-based trapdoor commitment sheme [5](IBTC for short) as another important tool in our construction.

Definition 2.5(IBTC scheme [5]) Let $k$ be complexity parameter, the non-interactive identity-based trapdoor commitment sheme IBTC $=(\mathrm{D}$, Setup, UKG, Cmt, Vf, FakeCmt, FakeDmt) is a group of P.P.T. algorithms, where $\mathrm{D}(k)$ generates $i d, \operatorname{Setup}(k)$ generates master public/secret-key pair ( $m p k, m s k$ ), $\mathrm{UKG}(m s k, i d)$ generates id's user private-key usk(id), $\operatorname{Cmt}(m p k, i d, M)$ generates message $M$ 's commitment/decommitment pair (cmt,dmt), $\operatorname{Vf}(m p k, i d, M, c m t, d m t)$ outputs 0 or 1 , verifying whether $c m t$ is $M$ 's commitment with respect to $i d$. These algorithms are consistant, i.e., for any $M$ :

$\mathrm{P}[(m p k, m s k) \leftarrow \operatorname{Setup}(k) ;(c m t, d m t) \leftarrow \operatorname{Cmt}(m p k, i d, M): \operatorname{Vf}(m p k, i d, M, c m t, d m t)=1]=1$

FakeCmt $(m p k, i d, u s k(i d))$ generates $(\overline{c m t}, \lambda), \operatorname{FakeDmt}(m p k, M, \lambda, \overline{c m t})$ generates $\bar{d}$ (w.l.o.g. $\lambda$ contains $i d \| u s k(i d)$ as one of its components so FakeDmt doesn't explicitly take $i d$ and $u s k(i d)$ as its input). A secure IBTC scheme has the following properties:

(1)Hiding: for any $i d$ and $M_{0}, M_{1},\left(c m t_{\mathrm{i}}, d m t_{\mathrm{i}}\right) \leftarrow \operatorname{Cmt}\left(m p k, i d, M_{\mathrm{i}}\right), \mathrm{i}=0,1$, then $c m t_{0} \approx{ }^{\text {P.P.T. }} \mathrm{cmt}_{1}$;

(2)Binding: for any P.P.T. algorithm $A$, the function $A d v_{I B T C, A}^{\text {binding }}(k) \equiv \mathrm{P}\left[(m p k, m s k) \leftarrow \operatorname{Setup}(k)\right.$; $\left(i d^{*}\right.$, $\left.c m t^{*}, M_{0} *, d_{0} *, M_{1} *, d_{1}^{*}\right) \leftarrow A^{\mathrm{UKG}(m s k, .)}(m p k): A$ doesn't query oracle-U(msk,.) with $i d^{*} \wedge M_{0} * \neq M_{1} *$ $\left.\wedge \mathrm{Vf}\left(m p k, i d^{*}, M_{0} *, c m t^{*}, d_{0}^{*}\right)=\mathrm{Vf}\left(m p k, i d^{*}, M_{1}^{*}, c m t^{*}, d_{1}^{*}\right)=1\right]$ is always negligible in $k$.

(3)Equivocability: For any P.P.T. algorithm $A=\left(A_{1}, A_{2}\right)$ the following experiment always has $\left|\mathrm{P}\left[b^{*}=b\right]-1 / 2\right|$ upper-bounded by a negligible function in $k$ :

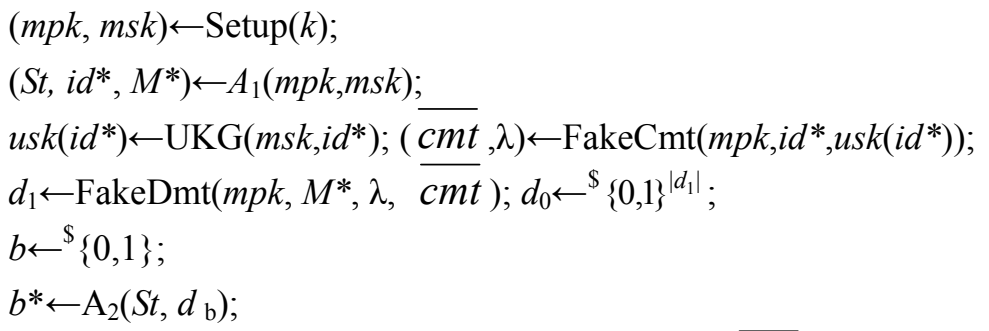

Note that equivocability implies $\operatorname{P}\left[\operatorname{Vf}\left(m p k, i d^{*}, M^{*}, \overline{c m t}, d_{1}^{*}\right)=1\right]>1-\gamma(k)$ where $\gamma(k)$ is a negligible function in $k$. [5] presented an efficient IBTC construction and proved its security.

\section{GENERAL CONSTRUCTION}

Now we present the formal consctrution of the real-world private equijoin protocol $\Psi . \mathrm{P}_{1}$ and $\mathrm{P}_{2}$ denote two real-world parties with private tables $\mathrm{X}_{1}$ and $\mathrm{X}_{2}$, each with attributes $w, x$ and $w, y$ respectively (more generally $x$ is the vector of all attributes in $\mathrm{X}_{1}$ othe than $w$, similar for $y$, but this is 
immaterial in our approach), $\mathrm{X}_{1}=\left\{\left(u_{1}, x_{1}\right), \ldots,\left(u_{N_{1}}, x_{N_{1}}\right)\right\}, \mathrm{X}_{2}=\left\{\left(v_{1}, y_{1}\right), \ldots,\left(v_{N_{2}}, y_{N_{1}}\right)\right\}$ where $u_{i}$ 's, $v_{j}$ 's are values of $w, x_{i}$ 's are values of $x$ and $y_{j}$ 's are values of $y$. $\Pi=(E S e t u p, U K G, E, D)$ is a id-selective ANO_CPA anonymous and id-selective IND_CPA data-private IBE scheme, $\Delta_{\text {Blind-UKG }}^{\Pi}$ is the real-world protocol for ח's user private-keys blind generation. In addition, we suppose a predefined bijective coding function $H$ maping strings or values (e.g., $x_{i}, y_{j}$ ) to $\Pi$ 's plaintexts, but for simplicity we always write $\mathrm{E}(m p k, a, x \mid y)$ instead of $\mathrm{E}(m p k, a, H(x \| y))$. IA-NMZPoK $(w: R(x, w)=1)$ denotes an IA-NMZPoK protocol for relation $R$ where $w$ is $x$ 's witness. TC $=(\mathrm{D}, \mathrm{TSetup}, \mathrm{UKG}, \mathrm{Cmt}, \mathrm{Vf}, \mathrm{FakeCmt}$, FakeDmt) is an IBTC scheme. $M_{0}$ is a (fixed) public common string and $\left|M_{0}\right|=\operatorname{poly}(k)$. $\Psi$ 's ACRS is $m p k_{\mathrm{TC}}\left\|m p k_{\Delta}\right\| m p k_{\mathrm{ZK}} \| M_{0}$ where $m p k_{\mathrm{TC}}, m p k_{\Delta}, m p k_{\mathrm{ZK}}$ are respectively TC's, $\Delta^{\Pi}{ }_{\text {Blind-UKG }}$ 's and an IA-NMZPoK protocol (see below)'s master public key. $\Psi$ works as follows. For intuition it is also presented in a figure where the IA-NMZPoK protocol's arrow points from the prover to its verifier.

\section{Equijoin Protocol $\Psi$ : General Construction}

(1) $\mathrm{P}_{1}$ computes $\Pi$ 's master public/secret-key $(m p k, m s k) \leftarrow \operatorname{ESetup}(k)$, for each $\left(u_{i}, x_{i}\right) \in \mathrm{X}_{1}\left(i=1, \ldots, \mathrm{N}_{1}\right)$ computes ciphertext $\xi_{i} \leftarrow \mathrm{E}\left(m p k, u_{i}, x_{i}|| M_{0} ; r_{i}\right)$ where $r_{i}$ is the independent randomness in each encryption, then computes $(c m t, d m t) \leftarrow \mathrm{Cmt}\left(m p k_{\mathrm{TC}}, \mathrm{P}_{2}, \xi_{1}\|\ldots\| \xi_{\mathrm{N} 1}\right)$ and sends $m p k \| c m t$ to $\mathrm{P}_{2}$.

(2) $\mathrm{P}_{1}$ and $\mathrm{P}_{2}$ run the protocol $\Delta{ }^{\Pi}{ }_{\text {Blind-UKG }}$ where $\mathrm{P}_{1}$ (as the key-generater) inputs (mpk,msk) and $\mathrm{P}_{2}$ (as

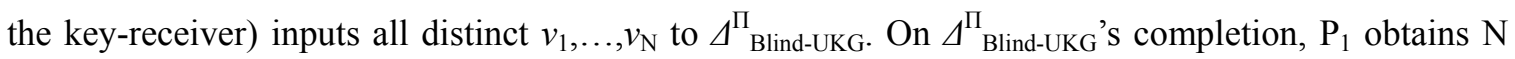
and $\mathrm{P}_{2}$ obtains $u s k\left(v_{1}\right), \ldots, u s k\left(v_{\mathrm{N}}\right)$ as the output.

(3) $\mathrm{P}_{1}$ sends $\xi_{1}\|\ldots\| \xi_{\mathrm{N} 1} \| d m t$ to $\mathrm{P}_{2}$.

(4) $\mathrm{P}_{2}$ verifies $\operatorname{Vf}\left(m p k_{\mathrm{TC}}, \mathrm{P}_{2}, \xi_{1}\|\ldots\| \xi_{\mathrm{N} 1}, c m t, d m t\right)=1$.

(5) $\mathrm{P}_{1}$ runs the protocol IA-NMZPoK $\left(\left(u_{i}, x_{i}, r_{i}\right): \xi_{i}=\mathrm{E}\left(m p k, u_{i}, x_{i}|| M_{0} ; r_{i}\right), i=1, \ldots, \mathrm{N}_{1}\right)$ as a prover with $\mathrm{P}_{2}$ as a verifier. On this IA-NMZPoK 's completion, $\mathrm{P}_{2}$ tries to decrypt each $\xi_{i}$ by all $u s k\left(v_{j}\right)$ 's it obtained in step 2 and everytime the output has suffix $M_{0}$, i.e., $\mathrm{D}\left(m p k, u s k\left(v_{j}\right), \xi_{i}\right)=x_{i} \| M_{0}$, it generates an entry $\left(v_{j}\right.$, $\left.x_{i}\right)$. All such entries constitute a temporary table $\mathrm{X}_{0}$, then $\mathrm{P}_{2}$ performs a local join $\mathrm{Y}_{0} \leftarrow \operatorname{Join}\left(w: \mathrm{X}_{0}, \mathrm{X}_{2}\right)$.

(6) $\mathrm{P}_{1}$ outputs $\mathrm{N}$ and $\mathrm{P}_{2}$ outputs $\mathrm{Y}_{0}$.

Note that $\mathrm{X}_{0}=\left\{\left(v_{j}, x_{i}\right) \in \mathrm{X}_{1}\right.$ : there exists $\xi_{i}$ s.t. $\left.\mathrm{D}\left(m p k, u s k\left(v_{j}\right), \xi_{i}\right)=x_{i}|| M_{0}\right\}$ and $\mathrm{D}\left(m p k, u s k\left(v_{j}\right), \xi_{i}\right)=$ $x_{i}|| M_{0}$ implies $u_{i}=v_{j}$ with negligible exception, so $\mathrm{Y}_{0}=\operatorname{Join}\left(w: \mathrm{X}_{1}, \mathrm{X}_{2}\right)$, i.e., $\Psi$ 's output is correct with only negligible exception probability.

$\Psi$ is atually a $\Delta^{\Pi}$ Blind-UKG -hybrid protocol and we require $\Delta^{\Pi}{ }_{\text {Blind-UKG }} \rightarrow{ }^{\mathrm{GUC}} F^{\Pi}{ }_{\text {Blind-UKG }}$ (definition 2.1). However, merely requiring $\Delta^{\Pi}{ }_{\text {Blind-UKG }} \rightarrow{ }^{\mathrm{GUC}} F^{\Pi}{ }_{\text {Blind-UKG }}$ cannot guarantee $\Psi$ 's GUC-security but only "half GUC-security" instead (i.e., the real adversary $A$ corrupting $\mathrm{P}_{1}$ can be completely simulated by an ideal adversary $S$ but this is not true when $A$ corrupts $\mathrm{P}_{2}$. Only data-privacy can be proved in the latter case). In order to make the real adversary completely simulatable in ideal-world, some additional

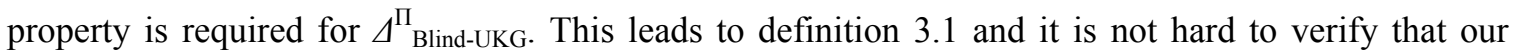
concrete construction of $\Delta^{\Pi}{ }_{\text {Blind-UKG }}$ in next section really satisfies it.

Definition 3.1(IBE's User Private-keys Blind Generation Protocol with Extractor) Given IBE scheme $\Pi=\left(\right.$ ESetup,UKG,E,D) and $\Delta^{\Pi}{ }_{\text {Blind-UKG }} \rightarrow{ }^{\mathrm{GUC}} F^{\Pi}{ }_{\text {Blind-UKG }}$, let $\mathrm{P}_{1}, \mathrm{P}_{2}$ be $\Delta^{\Pi}{ }_{\text {Blind-UKG }}$ 's parties where $\mathrm{P}_{2}$ provides user-id $a$ and obtains usk(a), $\mathrm{P}_{1}$ owns $m s k$ and (blindly) gernates $u s k(a)$ for $\mathrm{P}_{2}$. This $\Delta^{\Pi}{ }_{\text {Blind-UKG is defined as extractable, if there exists P.P.T. algorithm Setup }}, \mathrm{UKG}_{\Delta}, \operatorname{Ext}_{\Delta}=\left(\mathrm{Ext}_{1}, \mathrm{Ext}_{2}\right)$ and a negligible function $\delta(k)$, called the error function, such that

(1) $\operatorname{Setup}_{\Delta}(k)$ generates the master public/secret-key pair $\left(m p k_{\Delta}, m s k_{\Delta}\right)$. 
(2) $\mathrm{UKG}_{\Delta}\left(m s k_{\Delta}, i d\right)$ outputs a trapdoor $u s k_{\Delta}\left(\mathrm{P}_{2}\right)$ when $i d=\mathrm{P}_{2}$ (key-receiver's identity) and outputs nothing otherwise.

(3) for any user-id $a$, honest $\mathrm{P}_{1}$ and any P.P.T. algorithm $A$, it is true that(via notations in subsection 2.3) $\operatorname{Ext}_{1}\left(u s k\left(\mathrm{P}_{2}\right)\right)$ outputs $(\sigma, \tau)$ such that

$$
\mathrm{P}\left[\operatorname{Ext}_{2}(m p k|| \tau ; A(a))_{[\sigma]}=a\right]>\mathrm{P}\left[A_{a}\left(m p k ; P_{1}(m p k, m s k)\right)_{[\sigma]}=\mathrm{UKG}(m s k, a)\right]-\delta(k)
$$

where ( $m p k, m s k)$ is $\Pi$ 's master public/secret-key owned by $\mathrm{P}_{1}(m p k$ is published).

We stress that all extractors in definition 2.3 and definition 3.1 are non-rewinding.

$$
\begin{aligned}
& \mathrm{P}_{1}\left(\mathrm{X}_{1}\right) \quad A C R S=m p k_{\mathrm{TC}}\left\|m p k_{\Delta}\right\| m p k_{\mathrm{ZK}} \| M_{0} \quad \mathrm{P}_{2}\left(\mathrm{X}_{2}\right) \\
& (m p k, m s k) \leftarrow \operatorname{ESetup}(k) \\
& \text { for each }\left(u_{i}, x_{i}\right) \in \mathrm{X}_{1} \text { do } \\
& \xi_{\mathrm{i}} \leftarrow \mathrm{E}\left(m p k, u_{i}, x_{i}|| M_{0} ; r_{i}\right) ; \\
& (c m t, d m t) \leftarrow \operatorname{Cmt}\left(m p k_{\mathrm{TC}}, \mathrm{P}_{2}, \xi_{1}\|\ldots\| \xi_{\mathrm{N} 1}\right) \text {; } \\
& m p k \| c m t \\
& \text { input } v_{1}\|\ldots\| v_{\mathrm{N}} \text { where } v_{j} \text { goes }
\end{aligned}
$$

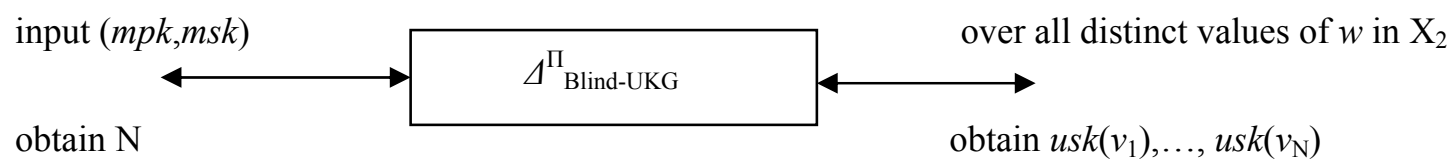

$$
\begin{aligned}
& \xi_{1}\|\ldots\| \xi_{\mathrm{N} 1} \| d m t \\
& \text { verify } \operatorname{Vf}\left(m p k_{\mathrm{TC}}, \mathrm{P}_{2}, \xi_{1}\|\ldots\| \xi_{\mathrm{N} 1}, c m t, d m t\right)=1 \\
& / * \mathrm{Vf} \text { is the IBTC-scheme TC's verification function.*/ } \\
& \underset{\operatorname{IA-NMZPoK}\left(\left(u_{i}, x_{i}, r_{i}\right): \xi_{i}=\mathrm{E}\left(m p k, u_{i}, x_{i}|| M_{0} ; r_{i}\right), i=1, \ldots, \mathrm{N}_{1}\right)}{\mathrm{X}_{0} \leftarrow\left\{\left(v_{j}, x_{i}\right)\right.} \text { : there exists } \xi_{i} \text { s.t. } \\
& \left.\mathrm{D}\left(m p k, u s k\left(v_{j}\right), \xi_{i}\right)=x_{i}|| M_{0}\right\} \\
& \operatorname{output}(\mathrm{N}) \quad \operatorname{output}\left(\mathrm{N}_{1} \| \operatorname{Join}\left(w: \mathrm{X}_{0}, \mathrm{X}_{2}\right)\right.
\end{aligned}
$$

Combining all the instantiations of subprotocols in this general construction (some presented in next section and Appendix D), it's easy to see that we can get a $\mathrm{O}(1)$ and $\mathrm{O}\left(\mathrm{N}_{1}+\mathrm{N}_{2}\right)$ message-complexity solution. The exact computation efficiency analysis can be only done for specific instantiations (e.g., that presented in next section) which is provided in the full version paper.

Theorem 3.1 Suppose that IBE scheme $\Pi=$ (ESetup,UKG,E,D) is both id-selective ANO_CPA anonymous and id-selective IND_CPA data-private, $\Delta^{\Pi}{ }_{\text {Blind-UKG }} \rightarrow{ }^{\mathrm{GUC}} F_{\text {Blind-UKG }}^{\Pi}$ with extractor $\operatorname{Ext}_{\Pi}=\left(\operatorname{Ext}_{\Pi, 1}, \operatorname{Ext}_{\Pi, 2}\right)$ and error function $\delta$ as in def.3.1, IA-NMZPoK $\left(\left(u_{i}, x_{i}, r_{i}\right): \xi_{i}=\mathrm{E}\left(m p k, u_{i}, x_{i}|| M_{0} ; r_{i}\right)\right.$, $\left.i=1, \ldots, \mathrm{N}_{1}\right)$ is an IA-NMZPoK protocol, TC $=(\mathrm{D}, \mathrm{TSetup}, \mathrm{UKG}, \mathrm{Cmt}, \mathrm{Vf}$, FakeCmt,FakeDmt) is an IBTC scheme, then $\Psi \rightarrow{ }^{\mathrm{GUC}} F_{\text {join }}$ assuming static corruptions.

The proof is in Appendix B.

\section{AN INSTANTIATION VIA BOYEN-WATERS IBE SCHEME}

Theorem 3.1 presents security conditions for the general construction $\Psi$, among which some are 
available in existing works, e.g., the commitment scheme can be directly borrowed from [5]. The subprotocols which require new efficient constructions are only IBE scheme's user private-keys generation protocol and the protocol IA-NMZPoK $\left((a, r): \xi=\mathrm{E}\left(m p k, a, M_{0} ; r\right)\right)$. In this section we present an efficient instantiation of $\Psi$ via Boyen-Waters IBE scheme. The related zero-knowledge protocol's construction is presented in Appendix D.

\subsection{Boyen-Waters IBE ${ }^{[3]}$}

Given an bilinear group pairing ensemble $\mathrm{J}=\left\{\left(p, \mathrm{G}_{1}, \mathrm{G}_{2}, e\right)\right\}_{k}$ where $\left|\mathrm{G}_{1}\right|=\left|\mathrm{G}_{2}\right|=p, p$ is $k$-bit prime number, $\mathrm{P} \in \mathrm{G}_{1}, e: \mathrm{G}_{1} \times \mathrm{G}_{1} \rightarrow \mathrm{G}_{2}$ is a non-degenerate pairing, Boyen-Waters IBE consists of

\section{$\operatorname{ESetup}(k)$}

$$
\begin{aligned}
& g, g_{0}, g_{1} \leftarrow{ }^{\$} \mathrm{G}_{1} ; \omega, t_{1}, t_{2}, t_{3}, t_{4} \leftarrow{ }^{\$} Z_{\mathrm{p}} ; \Omega \leftarrow e(g, g)^{t_{1} t_{2} \omega} ; \\
& v_{1} \leftarrow g^{t 1} ; v_{2} \leftarrow g^{t 2} ; v_{3} \leftarrow g^{t 3} ; v_{4} \leftarrow g^{t 4} ; \\
& m p k \leftarrow\left(\mathrm{G}_{1}, \mathrm{G}_{2}, p, e, \Omega, g, g_{0}, g_{1}, v_{1}, v_{2}, v_{3}, v_{4}\right) ; \\
& m s k \leftarrow\left(\omega, t_{1}, t_{2}, t_{3}, t_{4}\right) ; \\
& \text { return ( } m p k, m s k) \text {; } \\
& r_{1}, r_{2} \leftarrow{ }^{\$} Z_{\mathrm{p}} ; \\
& u s k(a) \leftarrow\left(g^{r_{1} t_{1} t_{2}+r_{2} t_{3} t_{4}}, g^{-\varpi t_{2}}\left(g_{0} g_{1}^{a}\right)^{-r_{1} t_{2}}, g^{-\varpi t_{1}}\left(g_{0} g_{1}^{a}\right)^{-r_{1} t_{1}},\left(g_{0} g_{1}^{a}\right)^{-r_{2} t_{4}},\left(g_{0} g_{1}^{a}\right)^{-r_{2} t_{3}}\right) \text {; } \\
& \text { return }(u s k(a)) \text {; }
\end{aligned}
$$

$\mathrm{UKG}(m s k, a), a \in Z_{\mathrm{p}}$ :

$\mathrm{E}(m p k, a, M), M \in \mathrm{G}_{2}$ :

$$
s, s_{1}, s_{2} \leftarrow^{\$} Z_{\mathrm{p}} ; \xi \leftarrow\left(\Omega^{\mathrm{s}} M,\left(g_{0} g_{1}{ }^{a}\right)^{s}, v_{1}{ }^{\mathrm{s}-\mathrm{s} 1}, v_{2}{ }^{\mathrm{s} 1}, v_{3}^{\mathrm{s}-\mathrm{s} 2}, v_{4}{ }^{\mathrm{s} 2}\right) ; \operatorname{return}(\xi) ;
$$

$\mathrm{D}\left(m p k, u s k(a),\left(\xi_{00}, \xi_{0}, \xi_{1}, \xi_{2}, \xi_{3}, \xi_{4}\right)\right)$ where $u s k(a) \equiv\left(d_{0}, d_{1}, d_{2}, d_{3}, d_{4}\right)$ :

$$
T \leftarrow e\left(d_{0}, \xi_{0}\right) e\left(d_{1}, \xi_{1}\right) e\left(d_{2}, \xi_{2}\right) e\left(d_{3}, \xi_{3}\right) e\left(d_{4}, \xi_{4}\right) ; \operatorname{return}\left(\xi_{00} T\right) ;
$$

[3] has proven that assuming the decisional bilinear Diffie-Hellman problem(D-BDHP)'s hardness on $\mathrm{J}$, this scheme is id-selective IND_CPA data-private; assuming the decisional linear problem(D-LP)'s hardness, this scheme is id-selective ANO_CPA anonymous. Notice that D-BDHP hardness implies D-LP's hardness, all the above consequences can be also obtained only under D-BDHP's hardness.

\subsection{User Private-Keys Blind Generation Protocol $\Delta_{B l i n d-U K G}^{\text {Boyen-Waters }}$ and Its GUC-Security}

For simplicity we only present how to blindly generate $u \operatorname{sk}(a)$ for a single user-id $a$. The generalization to blindly generating $u s k\left(a_{1}\right)\|\ldots\| u s k\left(a_{\mathrm{N}}\right)$ for multiple user-id's $a_{1}\|\ldots\| a_{\mathrm{N}}$ is trival and still constant-round, though the total message-complexity is linearly increased.

The two parties are $\mathrm{P}_{1}$ (with private input $m s k$ ) and $\mathrm{P}_{2}$ (with private input $a$ ). Both parties have the common input $m p k$ where ( $m p k, m s k$ ) are generated by IBE scheme's ESetup( $k$ ) (usually $m s k$ per se is the randomness in ESetup so we use a simplified notation $m p k \leftarrow \mathrm{ESetup}(m s k)$ hereafter). $\Delta_{\text {Blind-UKG }}^{\text {Boy-Water }}$ has two IA-NMZPoK subprotocols (see below) which ACRS's are denoted as $m p k_{\mathrm{ZK}, \mathrm{II}}$ and $m p k_{\mathrm{ZK}, \mathrm{III}}$. $\Delta_{\text {Blind-UKG }}^{\text {Boyen-Waters }}$ is in ACRS model which ACRS is $m p k_{\mathrm{ZK}, \mathrm{II}}|| m p k_{\mathrm{ZK}, \mathrm{III}} \cdot \Delta_{\text {Blind-UKG }}^{\text {Boyen-Waters }}$ works as follows: 
(1) $\mathrm{P}_{1}$ runs a protocol IA-NMZPoK $(m s k$ : $m p k=\operatorname{ESetup}(m s k))$ as a prover with $\mathrm{P}_{2}$ as a verifier, where the meaning of the notation IA-NMZPoK $(m s k$ : $m p k=\operatorname{ESetup}(m s k))$ follows section 3. Denote this protocol as IA-NMZPoK $\mathrm{II}_{\mathrm{II}}$.

(2) $\mathrm{P}_{2}$ selects $r_{1}, r_{2}, y_{1}, y_{2}, y_{3}, y_{4}$ at random, computes $U_{i} \leftarrow g^{r_{i}}, V_{i} \leftarrow\left(g_{0} g_{1}^{a}\right)^{-r_{i}}$ for $i=1,2$ and $h_{j} \leftarrow g^{y_{j}} g_{1}^{a}$ for $j=1,2,3,4$, sends $U_{1}\left\|U_{2}\right\| V_{1}\left\|V_{2}\right\| h_{1}\left\|h_{2}\right\| h_{3} \| h_{4}$ to $\mathrm{P}_{1}$. Then $\mathrm{P}_{2}$ runs the protocol

IA-NMZPoK $\left(\left(a, r_{1}, r_{2}, y_{1}, y_{2}, y_{3}, y_{4}\right): \wedge_{\mathrm{i}=1,2} U_{\mathrm{i}}=g^{r_{i}} \wedge_{\mathrm{i}=1,2} V_{\mathrm{i}}=\left(g_{0} g_{1}^{a}\right)^{-r_{i}} \wedge_{\mathrm{j}=1,2,3,4} h_{\mathrm{j}}=g^{y_{j}} g_{1}^{a}\right)$ as a prover with $\mathrm{P}_{1}$ as a verifier. Denote this protocol as IA-NMZPoK $\mathrm{III}_{\text {II }}$.

(3) $\mathrm{P}_{1}$ selects $\sigma, r_{1}{ }^{\prime}, r_{2}{ }^{\prime}$ at random, computes $d_{0} \leftarrow\left(g^{r_{1}^{\prime}} U_{1}^{\sigma}\right)^{t_{1} t_{2}}\left(g^{r_{2}^{\prime}} U_{2}^{\sigma}\right)^{t_{3} t_{4}} ; d_{1}{ }^{\prime} \leftarrow g^{-\sigma t_{2}}\left(h_{1} g_{0}\right)^{-r^{\prime} t_{2}} V_{1}^{\sigma t_{2}}$; $d_{1}{ }^{\prime \prime} \leftarrow g^{r_{1}^{\prime} t_{2}} ; \quad d_{2}^{\prime} \leftarrow g^{-\infty t_{1}}\left(h_{2} g_{0}\right)^{-r_{1}^{\prime} t_{1}} V_{1}^{\sigma t_{1}} ; d_{2}^{\prime \prime} \leftarrow g^{r_{1}^{\prime} t_{1}} ; d_{3}{ }^{\prime} \leftarrow\left(h_{3} g_{0}\right)^{-r_{2}^{\prime} t_{4}} V_{2}^{\sigma t_{4}} ; d_{3}{ }^{\prime \prime} \leftarrow g^{r_{2} t_{4}} ;$ $d_{4}^{\prime} \leftarrow\left(h_{4} g_{0}\right)^{-r_{2}^{\prime} t_{3}} V_{2}^{\sigma t_{3}} ; d_{4}^{\prime \prime} \leftarrow g^{r_{2} t_{3}}$ and sends $d_{0}\left\|d_{1^{\prime}}{ }^{\prime}\right\| d_{1}{ }^{\prime \prime}\left\|d_{2}^{\prime}\right\| d_{2}^{\prime \prime}\left\|d_{3^{\prime}}{ }^{\prime}\right\| d_{3}{ }^{\prime \prime}\left\|d_{4}^{\prime}\right\| d_{4} "$ to $\mathrm{P}_{2}$.

(4) $\mathrm{P}_{2}$ computes $d_{j} \leftarrow d_{j}^{\prime} d_{j}^{\prime \prime y_{j}}, j=1,2,3,4$ and outputs $\left(d_{0}, d_{1}, d_{2}, d_{3}, d_{4}\right)$.

For intuition the protocol is presented in the figure below where IA-NMZPoKs' arrows point from zero-knowledge's prover to its verifier.

$$
\begin{aligned}
& A C R S=m p k_{\mathrm{ZK}, \mathrm{II}}|| m p k_{\mathrm{ZK}, \mathrm{III}} \\
& \mathrm{P}_{1}(m p k, m s k) \quad \mathrm{P}_{2}(m p k, a) \\
& \text { IA-NMZPoK } \mathrm{II}_{\mathrm{II}}(m s k: m p k=\operatorname{Setup}(m s k)) \quad \text { select } r_{1}, r_{2}, y_{1}, y_{2}, y_{3}, y_{4} \text { at random; } \\
& U_{i} \leftarrow g^{r_{i}}, V_{i} \leftarrow\left(g_{0} g_{1}^{a}\right)^{-r_{i}}, i=1,2 \\
& h_{j} \leftarrow g^{y_{j}} g_{1}^{a}, \mathrm{j}=1,2,3,4 \\
& U_{1}\left\|U_{2}\right\| V_{1}\left\|V_{2}\right\| h_{1}\left\|h_{2}\right\| h_{3} \| h_{4}
\end{aligned}
$$$$
\text { IA-NMZPoK }{ }_{\mathrm{III}}\left(\left(a, r_{1}, r_{2}, y_{1}, y_{2}, y_{3}, y_{4}\right): \wedge_{\mathrm{i}=1,2} U_{i}=g^{r_{i}} \wedge_{\mathrm{i}=1,2} V_{i}=\left(g_{0} g_{1}^{a}\right)^{-r_{i}} \wedge_{j=1,2,3,4} h_{\mathrm{j}}=g^{y_{j}} g_{1}^{a}\right)
$$$$
\text { select } \sigma, r_{1}{ }^{\prime}, r_{2} \text { at random; }
$$

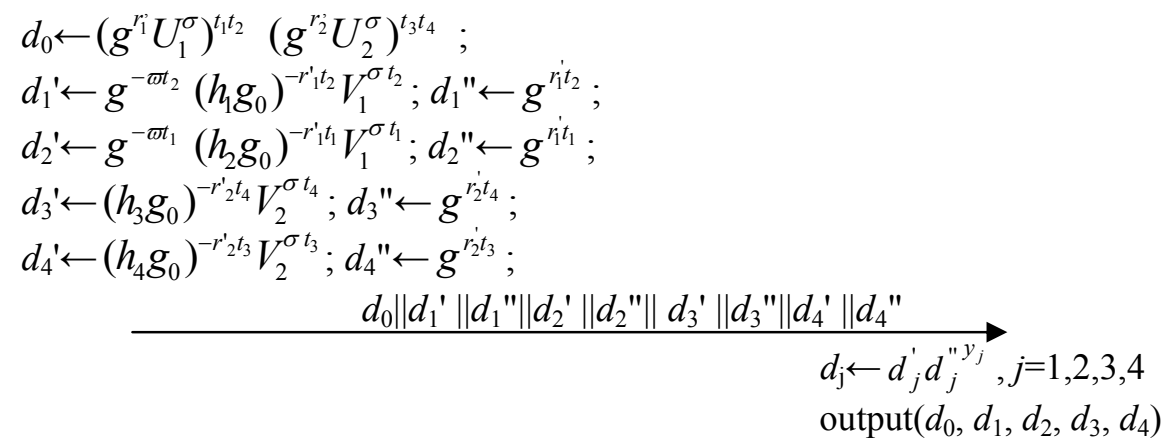

It's easy to show by direct calculation that $\mathrm{P}_{2}$ outputs the correct $u s k(a)=\left(d_{0}, d_{1}, d_{2}, d_{3}, d_{4}\right)$ where $d_{0}$ $=g^{\left(r_{1}{ }^{\prime}+r_{1} \sigma\right) t_{1} t_{2}+\left(r_{2}{ }^{\prime}+r_{2} \sigma\right) t_{3} t_{4}}, \quad d_{1}=g^{-\varpi t_{2}}\left(g_{0} g_{1}{ }^{a}\right)^{-\left(r_{1}{ }^{\prime}+r_{1} \sigma\right) t_{2}}, d_{2}=g^{-\varpi t_{1}}\left(g_{0} g_{1}{ }^{a}\right)^{-\left(r_{1}{ }^{\prime}+r_{1} \sigma\right) t_{1}}, d_{3}=$ $\left(g_{0} g_{1}{ }^{a}\right)^{-\left(r_{2}{ }^{\prime}+r_{2} \sigma\right) t_{4}}, d_{4}=\left(g_{0} g_{1}{ }^{a}\right)^{-\left(r_{2}{ }^{\prime}+r_{2} \sigma\right) t_{3}}$. Regarding security, we have

Theorem 4.1 Suppose the bilinear group pairing J has D-BDHP hardness, both IA-NMZPoK $K_{\mathrm{II}}$ and $I A-N M Z P o K_{\text {III }}$ are identity-augmented non-malleable zero-knowledge proof protocols for specific relations described in the above, then $\Delta_{\text {Blind-UKG }}^{\text {Boyen-Waters }} \rightarrow{ }^{\mathrm{GUC}} F_{\text {Blind-UKG }}^{\text {Boyen-Wats }}$ assuming static corruptions and $\Delta_{\text {Blind-UKG }}^{\text {Boyen-Waters }} \quad$ satisfies def. 3.1.

Appendix C gives the proof and Appendix D has the related IA-NMZPoK protocol's construction. 


\section{REFERENCES}

[1] M.Abdalla, M.Bellare, D.Catalano et al. Searchable Encryption Revisited: Consistency Properties, Relation to Anonymous IBE and Extensions. In Crypto'05, LNCS Vol. 3621, 205-222, 2005.

[2] I.Blake, V.Kolenilkov. Conditional Encrypted Mapping and Comparing Encrypted Numbers, In Proc. Financial Cryptography, 2006.

[3] X.Boyen, B.Waters. Anonymous Hierarchical Identity-based Encryption without Random Oracles, Crypto'06, LNCS Vol.4117, 290-307, 2006.

[4] R.Canetti, Universally Composable Security: a New Paradgim for Cryptographic Protocols, $42^{\text {nd }}$ Annual Symposium on foundations of computer Science, IEEE Computer Society, 136-145, 2001.Updated in 2005, eArchive Cryptology 2001/067.

[5] R.Canetti, Y.Dodis, R.Pass et al Universally Composable Security with Global-Setup, TCC'07, LCNS Vol.4392, 61-85, 2007. Full version available at eArchive Cryptology 2007.

[6] Y.Dodis, V.Shoup, S.Walfish Efficient Construction of Composable Commitment and Zero-Knowledge Proofs, Proc. Crypt'08, 2008.

[7] H.Garacia-Molina, J.Ullman, J.Widom Database System Implementation, Prentice-Hall, Inc.,2001.

[8] J.Garay, P..MacKenzie, K.Yang Strengthening Zero-Knowledge Protocols using Signatures, Proc. Eurocrypt, LNCS Vol.2656, 177-194, 2003.

[9] H. Hacigumus, B.Iyer, C.Li, S.Mehrotra Executing SQL over Encrypted Data in the Database-Service-Provider Model. In ACM SIGMOD'06, 96-107, 2006.

[10] C.Hazay, Y.Lindell Efficient Protocols for Set Intersection and Pattern Matching with Security against Malicious and Covert Adversaries, Proc. CT-RSA'08, 2008.

[11] J.Katz, A.Sahai, B.Waters. Predicate Encrypted Supporting Disjunctions, Polynomial Equations and Inner Products, In Proc. Eurocrypt'08, 146-162, 2008

[12] Y.Lindell, B.Pinkas. Privacy Preserving Data Mining. Journal of Cryptography 15,3(2002), 177-206.

[13] L.Kissner, D.Song Private-Preserving Set Operations, Crypto’05, LNCS Vol.3621, 241-257, 2005.

[14] P.MacKenzie, K.Yang On Simulation-Sound Trapdoor Commitments, In Proc. Eurocrypt'04, LNCS Vol.3027, 382-400, 2004.

[15] M.T.Ozsu, P.Valduriez. Principles of Distributed Database Systems, Prentice-Hall, Inc., 1999

[16] E.Shi, J.Bethencourt, H.Chen, D.X.Song, A.Perrig. Multi-dimensional Range Quries over Encrypted Data. In IEEE Symposium on Security and Privacy, 2007.

[17] B.Waters. Ciphertext-Policy Attribute-Based Encryption: An Expressive, Efficient and Provabley Secure Realization, eArchive 2008/304. 


\section{APPENDIX.A ACRS MODEL}

Recently [5] improves and generalizes the early UC-theory proposed in [4] to make a more general, realistic and strictly stronger security notion. The universal composition theorem is still true in this paradigm, however, the pre-setup needs to be strictly enhanced. In GUC paradigm the CRS model is insufficient to implement general cryptographic functionalities, instead we need a new pre-setup model called ACRS(augmented common reference string) model. This pre-setup can be performed via a shared functionality $\bar{G}_{a c r s}^{\text {Setu }, U K G}$ with two parameter functions Setup and UKG similar to IBE scheme's master public/secret-key generator and its user private-key generator. $\bar{G}_{\text {acrs }}^{\text {Setup } U K G}$,s program is [5]:

Initialization Phase: compute ( $m p k, m s k) \leftarrow \operatorname{Setup}(k)$ and store ( $m p k, m s k)$;

Running Phase: on receiving message ("CRS request", $\mathrm{P}_{i}$ ) from any party $\mathrm{P}_{i}$, response (“ACRS", mpk) to $\mathrm{P}_{i}$ and the adversary $S$;

On receiving message ("Retrieve",sid, $\mathrm{P}_{i}$ ) from a corrupt party $\mathrm{P}_{i}$, compute usk $\left(\mathrm{P}_{i}\right) \leftarrow \mathrm{UKG}\left(m s k, \mathrm{P}_{i}\right)$ and return the message ("Private-key", sid, usk $\left.\left(\mathrm{P}_{i}\right)\right)$ to $\mathrm{P}_{i} ;$ if $\mathrm{P}_{i}$ is not a corrupt party, response nothing.

\section{APPENDIX.B PROOF OF THEOREM 3.1}

We prove the GUC-security in two cases that the real-world adversary $A$ corrupts $\mathrm{P}_{1}$ or $\mathrm{P}_{2}$ respectively. Below $\mathrm{P}_{1} *$ and $\mathrm{P}_{2} *$ stand for $\mathrm{P}_{1}$ and $\mathrm{P}_{2}$ 's respective counterparts in ideal-world.

All parties are assumed to be initialized with a copy of the common reference string $A C R S$, i.e., the concatenation of TC's master public-key $m p k_{\mathrm{TC}}, \Delta^{\Pi}{ }_{\text {Blind-UKG }}$ 's $m p k_{\Delta}$, the IA-NMZPoK protocol's $m p k_{\mathrm{ZK}}$ and $M_{0}$, generated by the pre-setup $\mathrm{G}_{\mathrm{ACRS}}$. For this $A C R S$, its $m s k=m s k_{\mathrm{TC}}\left\|m s k_{\Delta}\right\| m s k_{\mathrm{ZK}}$ and $\mathrm{UKG}(m s k, i d)$ responses with $u s k(i d)=u s k_{\mathrm{TC}}(i d)\left\|u s k_{\Delta}(i d)\right\| u s k_{Z K}(i d)$ where $u s k_{\mathrm{TC}}(i d), u_{s} k_{\Delta}(i d)$ and $u s k_{\mathrm{ZK}}(i d)$ are respectively TC's, $\Delta_{\text {Blind-UKG's }}^{\Pi}$ and the IA-NMZPoK protocol's user private-keys corresponding to $i d \in\left\{\mathrm{P}_{1}, \mathrm{P}_{2}\right\}$.

(1) $A$ corrupts $\mathrm{P}_{1}$ : for simplicity we first make the proof in $F^{\Pi}{ }_{\text {Blind-UKG }}-$ hybrid model and then complete the proof by generalized universal composition theorem. Let $\mathrm{X}_{1}=\left\{\left(u_{1}{ }^{*}, x_{1} *\right), \ldots,\left(u_{\mathrm{N} 1}{ }^{*}, x_{\mathrm{N} 1}{ }^{*}\right)\right\}$ be $A$ 's(i.e., $\mathrm{P}_{1}$ 's) own table, $\mathrm{X}_{2}=\left\{\left(v_{1} *, y_{1} *\right), \ldots,\left(v_{\mathrm{N} 1} *, y_{\mathrm{N} 2} *\right)\right\}$ be $\mathrm{P}_{2}{ }^{*}$ 's own table. We need to construct an ideal adversary $S_{1}$ who corrupts $\mathrm{P}_{1}{ }^{*}$, runs $A$ as a black-box and simulates the real-world honest party $\mathrm{P}_{2}$ to interact with $A$ :

On receiving the message (sid,"input", $\left.\mathrm{N}_{2}\right)$ from $F_{\text {Join }}, S_{1}$ gets $u s k\left(\mathrm{P}_{1}\right)$ by querying the shared functionality $\mathrm{G}_{\mathrm{ACRS}}$ with ("retrieve",sid, $\mathrm{P}_{1}$ ) where $u s k\left(\mathrm{P}_{1}\right)=u s k_{\mathrm{TC}}\left(\mathrm{P}_{1}\right)\left\|u s k_{\Delta}\left(\mathrm{P}_{1}\right)\right\| u s k_{\mathrm{ZK}}\left(\mathrm{P}_{1}\right)$ ), computes $(\sigma, s, \tau) \leftarrow \mathrm{IA}-\mathrm{NMZPoK}:: \operatorname{Ext}_{1}\left(u s k_{Z K}\left(\mathrm{P}_{1}\right)\right)($ to avoid ambiguity, we use $\Gamma:: f$ to represent a protocol $\Gamma$ 's algorithm $f)$, generates $\mathrm{N}_{2}$ entries $\left(v_{1}, y_{1}\right), \ldots,\left(v_{\mathrm{N} 2}, y_{\mathrm{N} 2}\right)$ at random and then starts $A$;

After $A$ sends the first message $m p k \| c m t, S_{1}$ interacts with $A$ as an honest key-receiver in model of $F_{\text {Blind-UKG }}^{\Pi}$ and obtains $u s k\left(v_{1}\right), \ldots, u s k\left(v_{\mathrm{N} 2}\right)$;

$S_{1}$ intercepts the message $\xi_{1}\|\ldots\| \xi_{\mathrm{N} 1} \| d m t$ sent from $A$, verifies whether $\operatorname{Vf}\left(m p k_{\mathrm{TC}}, \mathrm{P}_{2}, \xi_{1}\|\ldots\| \xi_{\mathrm{N} 1}\right.$, $c m t, d m t)=1$ and then participates in protocol IA-NMZPoK $\left(\left(u^{*}{ }_{i}, x_{i}{ }_{i}, r_{i}\right): \xi_{i}=\mathrm{E}\left(m p k, u_{i}{ }^{*}, x^{*}{ }_{i} \| M_{0} ; r_{i}\right)\right.$, $i=1, \ldots, \mathrm{N}_{1}$ as a verifier calling the knowledge extractor IA-NMZPoK::Ext $\operatorname{Ex}_{2}(\tau)$ to extract the witness $\left(u_{i}^{*}, x_{i}{ }^{*}, r_{i}\right), \mathrm{i}=1, \ldots, \mathrm{N}_{1}$ (in fact only $u_{i}^{*}$ 's and $x_{i}{ }^{*}$ 's are needed in this proof); 
$S_{1}$ sends the message (sid, "input", $\left\{\left(u^{*}{ }_{1}, x_{1} *\right), \ldots,\left(u_{\mathrm{N} 1} *, x_{\mathrm{N} 1}\right)\right\}$ to $F_{\text {Join }}$, then outputs whatever $A$ outputs to the environment.

Let $\operatorname{tr}\left(A, S_{1}\right)$ denote the transcripts due to the interaction between $S_{1}$ and $A, \operatorname{tr}^{\mu}\left(A, \mathrm{P}_{2}\left(\mathrm{X}_{2}\right)\right)$ denote the transcripts due to the interaction between $A$ and $\mathrm{P}_{2}\left(\mathrm{X}_{2}\right)$ in the real-world protocol $\Psi\left(\mathrm{P}_{2}\left(\mathrm{X}_{2}\right)\right.$ means the real-world party possessing the same private table $\mathrm{X}_{2}$ as $\mathrm{P}_{2}{ }^{*}$ ). From $A$ 's perspective, the difference between $\operatorname{tr}\left(A, S_{1}\right)$ and $\operatorname{tr}^{\psi}\left(A, \mathrm{P}_{2}\left(\mathrm{X}_{2}\right)\right)$ is that the former provides $F^{\Pi}{ }_{\text {Blind-UKG }}$ with $\left\{v_{1}, \ldots, v_{\mathrm{N} 2}\right\}$ as the input, the latter provides $F^{\Pi}$ Blind-UKG with $\left\{v_{1}{ }^{*}, \ldots, v_{\mathrm{N} 2}{ }^{*}\right\}$, but according to $F_{\text {Blind-UKG }}^{\Pi}$ 's specification $A$ knows

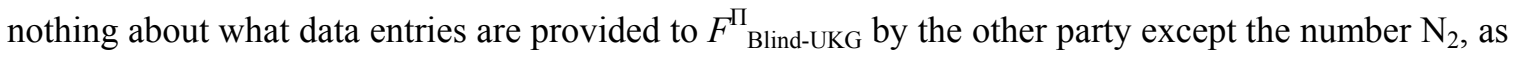
a result, $\operatorname{tr}\left(A, \mathrm{~S}_{1}\right) \approx \operatorname{tr}^{\psi}\left(A, \mathrm{P}_{2}\left(\mathrm{X}_{2}\right)\right)$ (perfectly indistinguishable) from $A$ 's perspective. In particular, the distribution of $A$ 's output due to interactions with $S_{1}$ is the same as that (in real-world protocol $\Psi$ ) due to interactions with $\mathrm{P}_{2}\left(\mathrm{X}_{2}\right)$. Let $\eta$ be IA-NMZPoK protocol's error function, $A d v_{T C}^{\text {binding }}$ be attacker's advantage against TC's binding property, all are negligible functions in $k$. It's not hard to show (by contradiction) that the probability with which $S_{1}$ correctly extracts all $A$ 's data-entries $\left(u^{*}{ }_{1}, x_{1}{ }^{*}\right), \ldots$, $\left(u^{*}{ }_{\mathrm{N} 1}, x_{\mathrm{N} 1} *\right)$ is greater than the probability $\mathrm{P}\left[\mathrm{P}_{2}\left(m p k\left\|\xi_{1}\right\| \ldots \| \xi_{\mathrm{N} 1} ; A\right)=1\right]-\mathrm{N}_{1}\left(\eta+A d v_{T C}^{\text {binding }}\right) \geq \mathrm{P}\left[\mathrm{P}_{2}\right.$ outputs $\left.\operatorname{Join}\left(w: \mathrm{X}_{1}, \mathrm{X}_{2}\right)\right]-\mathrm{N}_{1}\left(\eta+A d v_{T C}^{\text {binding }}\right)$, therefore, the difference between the probability with which $\mathrm{P}_{2} *\left(\mathrm{X}_{2}\right)$ outputs $\operatorname{Join}\left(w: \mathrm{X}_{1}, \mathrm{X}_{2}\right)$ under the ideal-world adversary $S_{1}$ 's attacks and the probability with which $\mathrm{P}_{2}\left(\mathrm{X}_{2}\right)$ outputs $\operatorname{Join}\left(w: \mathrm{X}_{1}, \mathrm{X}_{2}\right)$ under the real-world adversay $A$ 's attacks against $\Psi$ is upper-bounded by $\mathrm{N}_{1}\left(\eta+A d v_{T C}^{\text {binding }}\right)$, also a negligible function in $k$. Combining all the above facts, for any P.P.T. environment $Z$ we have $\operatorname{output}_{\mathrm{Z}}(\psi, A) \approx^{\mathrm{PPT}} \operatorname{output}_{\mathrm{Z}}\left(F_{\text {Join }}, S_{1}\right)$, i.e., $\Psi \rightarrow{ }^{\mathrm{GUC}} F_{\text {Join, }}$, in $F^{\Pi}{ }_{\text {Blind-UKG }}$-hybrid model.

Now replace the ideal functionality $F^{\Pi}{ }_{\text {Blind-UKG }}$ with $\Delta^{\Pi}{ }_{\text {Blind-UKG }}$ in $\Psi$. By what is just proved, the assumption $\Delta^{\Pi}{ }_{\text {Blind-UKG }} \rightarrow{ }^{\text {GUC }} F^{\Pi}{ }_{\text {Blind-UKG }}$ and the GUC-theorem, we still have the GUC-emulation consequence. In addition, it's not hard to estimate $S_{1}$ 's time complexity $\mathrm{T}_{S 1}=\mathrm{T}_{A}+\mathrm{O}\left(\mathrm{N}_{2}+\mathrm{N}_{1} \mathrm{~T}_{e}\right)$ where $\mathrm{T}_{A}$ and $\mathrm{T}_{e}$ are $A$ 's and the knowledge extractor's computation time.

(2) $A$ corrupts $\mathrm{P}_{2}$ : Denote $A$ 's(i.e., $\mathrm{P}_{2}$ 's $)$ own table as $\mathrm{X}_{2}=\left\{\left(v^{*}, y^{*}\right), \ldots,\left(v^{*}{ }_{\mathrm{N} 2}, y^{*}{ }_{\mathrm{N} 2}\right)\right\}, \mathrm{P}_{1}{ }^{*}$ 's own table as $\mathrm{X}_{1}=\left\{\left(u_{1}^{*}, x^{*}\right), \ldots,\left(\left(u^{*}{ }_{\mathrm{N} 1}, x^{*}{ }_{\mathrm{N} 1}\right)\right\}\right.$, we need to construct an ideal adversary $S_{2} . S_{2}$ corrupts $\mathrm{P}_{2}{ }^{*}$, gets usk $\left(\mathrm{P}_{2}\right)$ by querying the pre-setup $\mathrm{G}_{\mathrm{ACRS}}$ with ("retrieve",sid, $\mathrm{P}_{2}$ ) where usk $\left(\mathrm{P}_{2}\right)=u s k_{\mathrm{TC}}\left(\mathrm{P}_{2}\right)\left\|u s k_{\Delta}\left(\mathrm{P}_{2}\right)\right\|$ $u_{\text {sk }}\left(\mathrm{P}_{2}\right)$, generates $(\sigma, s) \leftarrow \mathrm{IA}-\mathrm{NMZPoK}:: \operatorname{Sim}_{1}\left(u s k_{\mathrm{ZK}}\left(\mathrm{P}_{2}\right)\right)$, runs $A$ as a black-box and simulates the real-world honest party $\mathrm{P}_{1}$ to interact with $A$ :

On receiving message (sid,"input", $\left.\mathrm{N}_{1}\right)$ from $F_{J o i n}, S_{2}$ generates $\left(u_{1}, x_{1}\right), \ldots,\left(u_{\mathrm{N} 1}, x_{\mathrm{N} 1}\right)$ at random, computes $(m p k, m s k) \leftarrow \operatorname{Setup}(k)$ and $\xi_{\mathrm{i}} \leftarrow \mathrm{E}\left(m p k, u_{\mathrm{i}}, x_{\mathrm{i}} \| M_{0} ; r_{\mathrm{i}}\right)$ for each $\left(u_{\mathrm{i}}, x_{\mathrm{i}}\right)$ where $r_{\mathrm{i}}$ is the independent randomness in each encryption, computes $\left(c m t^{0}, \lambda\right) \leftarrow \mathrm{FakeCmt}\left(m p k_{\mathrm{TC}}, \mathrm{P}_{2}, u s k_{\mathrm{TC}}\left(\mathrm{P}_{2}\right)\right)$, starts $A$ and sends the message $m p k \| c m t^{0}$ to $A$;

$S_{2}$ interacts with $A$ as the user private-key generator in $\Delta^{\Pi}{ }_{\text {Blind-UKG }}$ and calls the extractor $\Delta^{\Pi}{ }_{\text {Blind-UKG }}: \operatorname{Ext}_{\Delta}\left(u s k_{\Delta}\left(\mathrm{P}_{2}\right)\right)$ to extract $v^{*}{ }_{1}, \ldots, v^{*}{ }_{\mathrm{N}}\left(\mathrm{N}\right.$ is the number of distinct $v_{\mathrm{i}}^{*}$ 's), generates $y_{1}, \ldots, y_{\mathrm{N}}$ at random, sends the message (sid, "input", $\mathrm{P}_{2}{ }^{*},\left\{\left(v^{*}{ }_{1}, y_{1}\right), \ldots,\left(v^{*}{ }_{\mathrm{N}}, y_{\mathrm{N}}\right)\right\}$ to $F_{\text {Join }}$;

$S_{2}$ sends the message (sid,"join", $\left.\mathrm{P}_{2}{ }^{*}\right)$ to $F_{J o i n}$ and gets the response $\left\{\left(u^{*}{ }_{j 1}, x^{*}{ }_{j 1}, y_{j 1}\right), \ldots,\left(u^{*}{ }_{j t}, x^{*}{ }_{j t}\right.\right.$, $\left.\left.y_{j t}\right)\right\}$ (i.e., the equijoin of $\mathrm{X}_{1}$ and $\left\{\left(v^{*}, y_{1}\right), \ldots,\left(v^{*}, y_{\mathrm{N}}\right)\right\}$, in particular this result implies that there are t- $v_{j}$ 's such that $\left.u^{*}{ }_{j 1}=v_{j 1}, \ldots, u_{j t}=v^{*}{ }_{j t}\right)$. To simplify the notation, denote this response table as $\left\{\left(u^{*}{ }_{1}, x^{*}{ }_{1}\right.\right.$, $\left.\left.y_{1}\right), \ldots,\left(u^{*}, x_{t}^{*}, y_{t}\right)\right\}$.

$\mathrm{S}_{2}$ computes $\xi^{*} \leftarrow \mathrm{E}\left(m p k, u_{\mathrm{i}}, x_{\mathrm{i}}|| M_{0} ; r_{\mathrm{i}}\right)$ where $r^{*_{\mathrm{i}}}$ 's are independent randomness for $\mathrm{i}=1, \ldots, t$, 
replaces arbitrary $\mathrm{t} \xi_{\mathrm{i}}$ 's with $\xi_{\mathrm{i}}{ }^{*}$ 's and keeps other $\mathrm{N}_{1}-\mathrm{t} \xi_{\mathrm{i}}$ 's unchanged, making a new sequence denoted as $\xi_{1}{ }_{1}\|\ldots\| \xi_{\mathrm{N} 1}$, computes $d m t^{0} \leftarrow \operatorname{FakeDmt}\left(m p k_{\mathrm{TC}}, \xi_{1}{ }_{1}\|\ldots\| \xi_{\mathrm{N} 1}, \lambda, c m t^{0}\right) . S_{2}$ sends the message $\xi_{1}{ }_{1}\|\ldots\| \xi^{{ }}{ }_{\mathrm{N} 1} \| d m t^{0}$ to $A$, interacts with $A$ by calling IA-NMZPoK:: $\operatorname{Sim}_{2}\left(\xi^{\star}{ }_{1}\|\ldots\| \xi_{\mathrm{N} 1}, s\right)$ where $\xi_{\mathrm{i}}{ }_{\mathrm{i}}=\mathrm{E}(m p k$, $\left.u_{\mathrm{i}}^{0}, x_{\mathrm{i}}^{0} \| M_{0} ; r_{\mathrm{i}}{ }_{\mathrm{i}}\right), \mathrm{i}=1, \ldots, \mathrm{N}_{1}, u_{\mathrm{i}}^{0}=u_{\mathrm{i}}=v^{*}{ }_{\mathrm{i}}$ and $x_{\mathrm{i}}^{0}=x_{\mathrm{i}}$ for $\mathrm{i}=1, \ldots, \mathrm{t}$ and $u_{\mathrm{i}}^{0}=u_{\mathrm{i}}, x_{\mathrm{i}}^{0}=x_{\mathrm{i}}$ for other $\mathrm{i}$ 's.

Finally $S_{2}$ outputs whatever $A$ outputs to the environment.

Let $\operatorname{tr}\left(S_{2}, A\right)$ denote the transcripts due to the interaction between $A$ and $S_{2}, \operatorname{tr}^{\Psi}\left(\mathrm{P}_{1}\left(\mathrm{X}_{1}\right), A\right)$ denote the transcripts due to the interaction between $A$ and the real-world party $\mathrm{P}_{1}\left(\mathrm{X}_{1}\right)$ (possessing the same table $\mathrm{X}_{1}=\left\{\left(u_{1}^{*}, x_{1} *\right), \ldots,\left(u_{\mathrm{N} 1} *, x_{\mathrm{N} 1}\right)\right\}$ as the ideal-world party $\mathrm{P}_{1} *$. From $A$ 's perspective, the differences between these two transcripts are: a)cmt in these two transcripts are respectively $c m t^{0}$ output by FakeCmt and $c m t$ output by $\operatorname{Cmt}\left(m p k_{\mathrm{TC}}, \mathrm{P}_{2}, \mathrm{E}\left(m p k, u_{1}{ }^{*}, x_{1}{ }^{*} \| M_{0} ; r_{1}\right)\|\ldots\| \mathrm{E}\left(m p k, u^{*}{ }_{\mathrm{N} 1}\right.\right.$, $\left.\left.\left.x^{*}{ }_{\mathrm{N} 1} \| M_{0} ; r_{\mathrm{N} 1}\right)\right) ; b\right) d m t$ in these two transcripts are $d m t^{0}$ output by FakeDmt and $d m t$ output by $\operatorname{Cmt}\left(m p k_{\mathrm{TC}}, \mathrm{P}_{2}, \mathrm{E}\left(m p k, u_{1}{ }^{*}, x_{1}{ }^{*} \| M_{0} ; r_{1}\right)\|\ldots\| \mathrm{E}\left(m p k, u^{*}{ }_{\mathrm{N} 1}, x^{*}{ }_{\mathrm{N} 1} \| M_{0} ; r_{\mathrm{N} 1}\right)\right)$ respectively $\left.c\right)$ Among the ciphertext sequence $\xi_{1}\|\ldots\| \xi_{\mathrm{N} 1}$ in these two transcripts, there are $t$ ciphertexts $\xi_{\mathrm{i}}$ having the same identity public-key $u^{*}{ }_{i}$ and the same plaintext $x_{i}{ }_{i} \| M_{0}$ but the remaining $\mathrm{N}_{1}$-t ciphertexts having different identity public-keys and plaintexts; $d$ ) there are $t$ IA-NMZPoK-witness' with the same $u_{\mathrm{i}}^{0}$ and $x^{0}$.

Because of TC's equivocation property, $(c m t, d m t)$ 's are P.P.T.-indistinguishable in both cases; because of IBE scheme's selective ANO_CPA anonymity and IND_CPA data-privacy, $\xi_{1}\|\ldots\| \xi_{\mathrm{N} 1} \| d m t$ in both cases are P.P.T.-indistinguishable (otherwise suppose they are P.P.T.-distinguishable with the difference $\delta \geq 1 /$ poly $(k)$, it's not hard to construct either a selective ANO_CPA or IND_CPA attacker against $\Pi$ with an advantage at least $\delta / \mathrm{N}_{1}$, contradicting with $\Pi$ 's either selective ANO_CPA anonymity or data-privacy). Now denote the ciphertext sequence $\xi_{1}\|\ldots\| \xi_{\mathrm{N} 1}$ in two cases as $\xi_{1}{ }^{(1)}\|\ldots\|$ $\xi_{\mathrm{N} 1}{ }^{(1)}$ and $\xi_{1}{ }^{(2)}\|\ldots\| \xi_{\mathrm{N} 1}{ }^{(2)}$ respectively, denote the transcripts in session of IA-NMZPoK as $\mathrm{IA}-\mathrm{NMZPoK}^{(1)}\left(=\operatorname{tr}_{\mathrm{S} 2, \mathrm{~A}}\left(m p k\left\|M_{0}\right\| \xi_{1}{ }^{(1)}\|\ldots\| \xi_{\mathrm{N} 1}{ }^{(1)}\right)\right)$ and $\left.\mathrm{IA}^{-N_{M Z P o K}}{ }^{(2)}\left(=\operatorname{tr}_{\mathrm{P} 1, \mathrm{~A}}\left(m p k\left\|M_{0}\right\| \xi_{1}{ }^{(2)}\|\ldots\|_{\mathrm{N} 1}{ }^{(2)}\right)\right)\right)$ respectively, by the above analysis we have $\xi_{1}{ }^{(1)}\|\ldots\| \xi_{\mathrm{N} 1}{ }^{(1)} \approx^{\mathrm{PPT}} \xi_{1}{ }^{(2)}\|\ldots\|_{\mathrm{N} 1}{ }^{(2)}$; furthermore, by IA-NMZPoK's zero-knowledge property we have

$$
\text { IA-NMZPoK }{ }^{(2)} \approx \text { PPT IA-NMZPoK:: } \operatorname{Sim}_{2}\left(\xi_{1}{ }^{(2)}\|\ldots\| \xi_{\mathrm{N} 1}{ }^{(2)}, s\right)
$$

and by $S_{2}$ 's construction we have so IA-NMZPoK $^{(1)} \approx^{\text {PPT }}$ IA-NMZPoK ${ }^{(2)}$.

$$
\text { IA-NMZPoK }{ }^{(1)}=\mathrm{IA}-\mathrm{NMZPoK}:: \operatorname{Sim}_{2}\left(\xi_{1}{ }^{(1)}\|\ldots\| \xi_{\mathrm{N} 1}{ }^{(1)}, s\right)
$$

As a result, the transcripts received by $A$ in both cases are P.P.T.-indistinguishable.

Let $\delta$ be $\Delta^{\Pi}$ Blind-UKG's extractor's error function(negligible in $k$ ), then the probability with which $S_{2}$ correctly extracts $A$ 's one data-item $y^{*}{ }_{\mathrm{i}}$ is at least $\mathrm{P}\left[A\left(m p k ; P_{1}(m p k, m s k)\right)=\mathrm{UKG}\left(m s k, y^{*}\right)\right]-\delta$, so the probability with which $S_{2}$ correctly extracts $A$ 's all values $v^{*}{ }_{1}, \ldots, v^{*}{ }_{\mathrm{N}}$ is at least $\mathrm{P}\left[A\left(m p k ; P_{1}(m p k\right.\right.$, $\left.\left.m s k))=\mathrm{UKG}\left(m s k, v_{\mathrm{i}}\right)\right) \mathrm{i}=1, \ldots, \mathrm{N}\right]-\mathrm{N}_{2} \delta \geq \mathrm{P}\left[\mathrm{P}_{2}\right.$ outputs $\left.\operatorname{Join}\left(w: \mathrm{X}_{1}, \mathrm{X}_{2}\right)\right]-\mathrm{N} \delta$. As a result, $S_{2}$ 's output is P.P.T.-indistinguishable from $A$ 's output in $\Psi$ with respect to the GUC-environment $Z$ with an error upper-bounded by $\mathrm{N}_{1}(k)\left(A d v_{\Pi}^{A N O_{-} C P A}(k)+A d v_{\Pi}^{I N D_{-} C P A}(k)\right)+\mathrm{N}_{2} \delta\left(\mathrm{N} \leq \mathrm{N}_{2}\right)$ which is negligible in $k$. Note that in both cases the other party $\mathrm{P}_{1} *\left(\mathrm{X}_{1}\right)$ and $\mathrm{P}_{1}\left(\mathrm{X}_{1}\right)$ always output the same $\mathrm{N}$, so we have the consequence that $\operatorname{output}_{\mathrm{Z}}(\psi, A) \approx^{\mathrm{PPT}} \operatorname{output}_{\mathrm{Z}}\left(F_{\text {Join }}, \mathrm{S}_{2}\right)$ and it's easy to estimate $S_{2}$ 's time-complexity $\mathrm{T}_{S 2}=\mathrm{T}_{A}+\mathrm{O}\left(\mathrm{N}_{1}+\mathrm{N}_{2} \mathrm{~T}_{e x t}\right)$ where $\mathrm{T}_{A}$ and $\mathrm{T}_{e x t}$ are $A$ 's and the extractor's computation-time.

By all the facts, we have $\Psi \rightarrow{ }^{\mathrm{GUC}} F_{\text {Join }}$. 


\section{APPENDIX.C PROOF OF THEOREM 4.1}

All parties are assumed to be initialized with a copy of the common reference string $A C R S$, i.e., the concatenation of the two IA-NMZPoK protocol's $m p k_{\mathrm{ZK}, \mathrm{II}}$ and $m p k_{\mathrm{ZK}, \mathrm{III}}$. For this $A C R S$, $m s k=m s k_{\mathrm{ZK}, \mathrm{II}} \mid m s k_{\mathrm{ZK}, \mathrm{III}}$ and $\mathrm{UKG}(m s k, i d)$ outputs $u s k(i d)=u s k_{\mathrm{ZK}, \mathrm{II}}(i d) \| u s k_{\mathrm{ZK}, \mathrm{III}}(i d)$ where $u s k_{\mathrm{ZK}, \mathrm{II}}(i d)$ and $u s k_{\mathrm{ZK}, \mathrm{III}}(i d)$ are respectively two IA-NMZPoK protocol's user private-keys corresponding to $i d \in\left\{\mathrm{P}_{1}\right.$, $\left.\mathrm{P}_{2}\right\}$.

At first it's easy to show there exists an identity extractor for $\Delta_{B \text { lind }-U K G}^{\text {Boyen }}$ to satisfy definition 3.1. In fact it is IA-NMZPoK $\mathrm{III}\left(\left(a, r_{1}, r_{2}, y_{1}, y_{2}, y_{3}, y_{4}\right): \wedge_{\mathrm{i}=1,2} U_{\mathrm{i}}=g^{r_{i}} \wedge_{\mathrm{i}=1,2} V_{\mathrm{i}}=\left(g_{0} g_{1}^{a}\right)^{-r_{i}} \wedge_{\mathrm{j}=1,2,3,4} h_{\mathrm{j}}=\right.$ $\left.g^{y_{j}} g_{1}^{a}\right)$ 's knowledge extractor for which the to-be-extracted witness is $a$.

Now we prove $\Delta_{B \text { Bind-UKG }}^{\text {Boyen-Wats }}$ 's GUC-security in two cases that the real-world adversary $A$ corrupts $\mathrm{P}_{1}$ or $\mathrm{P}_{2}$ respectively. Below $\mathrm{P}_{1} *$ and $\mathrm{P}_{2} *$ stand for $\mathrm{P}_{1}$ and $\mathrm{P}_{2}$ 's respective counterparts in ideal-world.

(1) $A$ corrupts $\mathrm{P}_{1}$ : Suppose $A$ 's(i.e., $\mathrm{P}_{1}$ 's) private input is $(m p k, m s k), \mathrm{P}_{2}{ }^{*}$ 's private input is $a^{*}$. we need to construct an ideal adversary $S_{1} . S_{1}$ corrupts the ideal-world party $\mathrm{P}_{1}^{*}$, gets $u s k\left(\mathrm{P}_{1}\right)$ by querying $\mathrm{G}_{\mathrm{ACRS}}$ with the message ("retrieve",sid, $\left.\mathrm{P}_{1}\right)$ where $u s k\left(\mathrm{P}_{1}\right)=u s k_{\mathrm{ZK}, \mathrm{II}}\left(\mathrm{P}_{1}\right)|| u s k_{\mathrm{ZK}, \mathrm{III}}\left(\mathrm{P}_{1}\right)$, computes $\left(\sigma_{\mathrm{II}}, S_{\mathrm{II}}, \tau\right) \leftarrow \mathrm{IA}-\mathrm{NMZPoK}_{\mathrm{II}}: \operatorname{Ext}_{1}\left(u s k_{\mathrm{ZK}, \mathrm{II}}\left(\mathrm{P}_{1}\right)\right)\left(\right.$ notice that $\mathrm{P}_{1}$ is the prover in protocol IA-NMZPoK $\left.\mathrm{II}_{\mathrm{II}}\right)$, runs $A$ as a black-box. $S_{1}$ simulates the real-world honest party $\mathrm{P}_{2}$ to interact with $A$ :

In session of IA-NMZPoK $(m s k$ : $m p k=\operatorname{ESetup}(m s k)), S_{1}$ interacts with $A$ as a verifier extracting $m s k$ via running IA-NMZPoK ${ }_{\mathrm{II}}: \operatorname{Ext}_{2}(\tau)$, sends message (sid, $m p k \| m s k$ ) to $F_{\text {Blind -UKG }}^{\text {Boyen-Waters }}$;

$S_{1}$ generates an user-id $a$ at random, follows $\mathrm{P}_{2}$ 's specification in section 4.2 to compute $U_{1}, U_{2}, V_{1}$, $V_{2}, h_{1}, h_{2}, h_{3}, h_{4}$, sends $\quad U_{1}\left\|U_{2}\right\| V_{1}\left\|V_{2}\right\| h_{1}\left\|h_{2}\right\| h_{3} \| h_{4} \quad$ to $A$, computes $\quad\left(\sigma_{\mathrm{III}}, S_{\mathrm{III}}\right) \leftarrow$ IA-NMZPoK ${ }_{\mathrm{III}}::$ $\operatorname{Sim}_{1}\left(u s k_{\mathrm{ZK}, \mathrm{III}}\left(\mathrm{P}_{1}\right)\right)$ (notice that $\mathrm{P}_{1}$ is the verifier in protocol IA-NMZPoK $\left.{ }_{\text {III }}\right)$ and sends IA-NMZPoK $\mathrm{K}_{\mathrm{III}}:$ : $\operatorname{Sim}_{2}\left(U_{1}\left\|U_{2}\right\| V_{1}\left\|V_{2}\right\| h_{1}\left\|h_{2}\right\| h_{3} \| h_{4}, S_{\text {III }}\right)$ to $A$.

$S_{1}$ outputs whatever $A$ outputs to the environment.

Denote the second-round message in $\Delta_{\text {Blind-UKG }}^{\text {Boyen-Waters }}$ 's specification (i.e., $U_{1}\left\|U_{2}\right\| V_{1}\left\|V_{2}\right\| h_{1}\left\|h_{2}\right\| h_{3} \| h_{4}$ ) as $W$. From $A$ 's perspective, the transcripts due to its interactions with $S_{1}$ and the transcripts due to its interactions with the real-world party $\mathrm{P}_{2}\left(a^{*}\right)\left(\mathrm{P}_{2}\left(a^{*}\right)\right.$ stands for party $\mathrm{P}_{2}$ possessing $a^{*}$, the same private input as the ideal-world party $\left.\mathrm{P}_{2}{ }^{*}\right)$ differs in: $\left.a\right) W$ depends on $a$ in the former case, denoted as $W(a)$, while it depends on $a^{*}$ in the latter case and denoted as $\left.W\left(a^{*}\right) ; b\right)$ IA-NMZPoK $\mathrm{III}_{\mathrm{II}}$ 's witness depends on $a$ in the former case while it depends on $a^{*}$ in the latter. The messages of subprotocol IA-NMZPoK $\mathrm{III}_{\mathrm{II}}$ in these two cases are respectively denoted as IA-NMZPoK $\mathrm{III}(a)$ and IA-NMZPoK $\mathrm{III}_{\mathrm{II}}\left(a^{*}\right)$.

Let $\mathrm{g}_{0} \equiv g^{\alpha}, \mathrm{g}_{1} \equiv g^{\alpha^{*}}$. Explicitly expand $W(a)$ 's expression to $g^{r_{1}}\left\|g^{r_{2}}\right\| g^{-(\alpha+a \beta) r_{1}} \| g^{-(\alpha+a \beta) r_{2}}$ $\left\|g^{y_{1}+a \beta}\right\| \ldots \| g^{y_{4}+a \beta}$ and $W\left(a^{*}\right)$ to a similar expression where $a, r_{1}, r_{2}, y_{1}, y_{2}, y_{3}, y_{4}, \alpha$ and $a^{*}, r_{1}{ }^{*}, r_{2}{ }^{*}$, $y_{1}{ }^{*}, y_{2}{ }^{*}, y_{3}^{*}, y_{4}{ }^{*}, \alpha^{*}$ are probabilistically independent and all are unknown to $A$, so $W(a) \approx W\left(a^{*}\right)$ (perfectly indistinguishable). Furthuremore, by IA-NMZPoK ${ }_{\text {III }}$ 's zero-knowledge property we have

$$
\text { IA-NMZPoK }{ }_{\text {III }}: \operatorname{Sim}_{2}\left(W\left(a^{*}\right), s_{\text {III }}\right) \approx^{\text {PPT }} \text { IA-NMZPoK }_{\text {III }}\left(a^{*}\right)
$$

and by $S_{1}$ 's construction we also have

$$
\text { IA-NMZPoK } \mathrm{III}_{\mathrm{II}}: \operatorname{Sim}_{2}\left(W(a), s_{\mathrm{III}}\right)=\mathrm{IA}-\mathrm{NMZPo} K_{\mathrm{III}}(a)
$$

so $\mathrm{IA}_{\mathrm{NMZPoK}}(a)=\mathrm{IA}_{\mathrm{III}}\left(\mathrm{NMZPoK}_{\mathrm{III}}:: \operatorname{Sim}_{2}\left(W(a), \mathrm{s}_{\mathrm{III}}\right) \approx \mathrm{IA}_{\mathrm{N}} \mathrm{NMZPoK}_{\mathrm{III}}:: \operatorname{Sim}_{2}\left(W\left(a^{*}\right), \mathrm{S}_{\mathrm{III}}\right) \approx^{\mathrm{PPT}}\right.$

IA-NMZPoK ${ }_{\text {III }}\left(a^{*}\right)$. As a result, from $A$ 's perspective the transcripts due to its interactions with $S_{1}$ is 
P.P.T.-indistinguishable from that due to its interactions with $\mathrm{P}_{2}\left(a^{*}\right)$, in particular, the output of $A$ due to its interactions with $S_{1}$ is P.P.T.-indistinguishable from its output due to its interactions with $\mathrm{P}_{2}\left(a^{*}\right)$ in $\Delta_{\text {Blind-UKG }}^{\text {Boyen-Water }}$.

Let $\eta_{\mathrm{II}}$ denote IA-NMZPoK II 's knowledge extractor's error function(a negligible function in $k$ ), then the probability with which $\mathrm{P}_{2}{ }_{2}\left(a^{*}\right)$ outputs $\Pi:: \mathrm{UKG}\left(m s k, a^{*}\right)$ under $S_{1}$ 's attacks is at least $P\left[\mathrm{P}_{2}\right.$ accepts $m p k$ as a valid master public-key]- $\eta_{\mathrm{II}}$, i.e., except for an probability upper-bounded by $\eta_{\mathrm{II}}$, $\mathrm{P}_{2}{ }_{2}\left(a^{*}\right)$ 's output under $S_{1}$ 's attacks is the same as $\mathrm{P}_{2}\left(a^{*}\right)$ 's output under $A$ 's attacks, in other words, for any P.P.T. environment $Z$ we have output $\left(\Delta_{\text {Blind-UKG }}^{\text {Boyen-Waters }}, A_{1}\right) \approx^{\text {PPT }}$ output $_{\mathrm{Z}}\left(F_{\text {Blind-UKG }}^{\text {Boyen-Water }}, S_{1}\right)$ and it's easy to estimate $S_{1}$ 's time-complexity $\mathrm{T}_{S 1}=\mathrm{T}_{A}+\mathrm{T}_{e I I}+\mathrm{O}(1)$ where $\mathrm{T}_{A}$ and $\mathrm{T}_{e I \mathrm{II}}$ are $A$ 's and Ext $\mathrm{III}_{2}$ 's computation-time.

(2) $A$ corrupts $\mathrm{P}_{2}$ : Let $a$ denote $A$ 's (i.e., $\mathrm{P}_{2}$ 's) private input, $\left(m p k^{*}, m s k^{*}\right)$ denote the ideal-world party $\mathrm{P}_{1}{ }^{*}$ 's input where $m p k^{*}=\left(\mathrm{G}_{1}, \mathrm{G}_{2}, p, e, \Omega^{*}, g, g_{0}, g_{1}, v_{1}{ }^{*}, v_{2}{ }^{*}, v_{3}{ }^{*}, v_{4}{ }^{*}\right)$ and $m s k^{*}=\left(\omega^{*}, t_{1}^{*}, t_{2}{ }^{*}, t_{3}{ }^{*}, t_{4}{ }^{*}\right)$. We need to construct an ideal-world adversary $S_{2}$ which corrupts $\mathrm{P}_{2}^{*}$, gets $u s k\left(\mathrm{P}_{2}\right)$ by querying $\mathrm{G}_{\mathrm{ACRS}}$ with the message ("retrieve", $\left.\mathrm{sid}, \mathrm{P}_{2}\right)$ where $u s k\left(\mathrm{P}_{2}\right)=u s k_{\mathrm{ZK}, \mathrm{II}}\left(\mathrm{P}_{2}\right) \| u s k_{\mathrm{ZK}, \mathrm{III}}\left(\mathrm{P}_{2}\right)$, runs $A$ as a black-box and simulates the honest real-world party $\mathrm{P}_{1}$ to interact with $A$ :

On receiving the message ( $\operatorname{sid}, m p k^{*}$ ) from $F_{\text {Blind-UKG }}^{\text {Boy-Wats }}, S_{2}$ generates $\omega, t_{1}, t_{2}, t_{3}, t_{4}$ at random and computes

$$
\begin{aligned}
& \Omega \leftarrow e(g, g)^{t_{1} t_{2} \omega} ; v_{1} \leftarrow g^{t 1} ; v_{2} \leftarrow g^{t 2} ; v_{3} \leftarrow g^{t 3} ; v_{4} \leftarrow g^{t 4} ; \\
& m p k \leftarrow\left(\mathrm{G}_{1}, \mathrm{G}_{2}, p, e, \Omega, g, g_{0}, g_{1}, v_{1}, v_{2}, v_{3}, v_{4}\right) ; \\
& m s k \leftarrow\left(\omega, t_{1}, t_{2}, t_{3}, t_{4}\right) ; \\
& \left(\sigma_{\mathrm{II}}, S_{\mathrm{II}}\right) \leftarrow \mathrm{IA}-\mathrm{IMZPoK}_{\mathrm{II}}: \operatorname{Sim}_{1}\left(u s k_{\mathrm{ZK}, \mathrm{II}}\left(\mathrm{P}_{2}\right)\right) ; \\
& \left(\sigma_{\mathrm{III}}, S_{\mathrm{III}}, \tau\right) \leftarrow \mathrm{IA}-\mathrm{NMZPoK}_{\mathrm{III}}: \operatorname{Ext}_{1}\left(u s k_{\mathrm{ZK}, \mathrm{III}}\left(\mathrm{P}_{2}\right)\right) ;
\end{aligned}
$$

Note that $\mathrm{P}_{2}$ is the verifier in protocol IA-NMZPoK $\mathrm{II}_{\text {II }}$ and prover in IA-NMZPoK $\mathrm{III}$.

$S_{2}$ starts $A$ and interacts with it by running IA-NMZPoK $\mathrm{II}_{\mathrm{II}}: \operatorname{Sim}_{2}\left(m p k, S_{\mathrm{II}}\right)$;

When $A$ sends $U_{1}\left\|U_{2}\right\| V_{1}\left\|V_{2}\right\| h_{1}\left\|h_{2}\right\| h_{3} \| h_{4}$ and then launches IA-NMZPoK ${ }_{\text {III }}\left(\left(a, r_{1}, r_{2}, y_{1}, y_{2}, y_{3}\right.\right.$, $\left.\left.y_{4}\right) \ldots\right), S_{2}$ participates the session as an verifier by running IA-NMZPoK $\mathrm{III}_{\mathrm{II}}: \operatorname{Ext}_{2}(\tau)$ to extract $\left(a, r_{1}, r_{2}\right.$, $\left.y_{1}, y_{2}, y_{3}, y_{4}\right)$ (in fact only $a$ is used below);

$S_{2}$ sends the message ( $\left.\operatorname{sid} \| 1, a\right)$ to $F_{\text {Blind-UKG }}^{\text {Boyen-Wats }}$ and gets the response $\left(\operatorname{sid} \| 1, \mathrm{UKG}\left(m s k^{*}, a\right)\right.$ ) where $\mathrm{UKG}\left(m s k^{*}, a\right) \equiv\left(d_{0}^{*}, d_{1}^{*}, d_{2}^{*}, d_{3}^{*}, d_{4}^{*}\right)$;

$S_{2}$ generates $d_{j}{ }^{\prime \prime}$ at random, computes $d_{j}{ }^{6} \leftarrow d_{j}^{*} / d_{j}{ }^{\prime \prime}{ }_{j}, j=1,2,3,4$, sends $d^{*}{ }_{0}\left\|d_{1}{ }^{\prime}\right\| d_{1}{ }^{\prime \prime}\left\|d_{2}{ }^{\prime}\right\| d_{2}{ }^{\prime \prime} \| d_{3}{ }^{\prime}$ $\left\|d_{3}{ }^{\prime \prime}|| d_{4}^{\prime}\right\| d_{4} "$ to $A$.

Now we prove that from $A$ 's perspective the transcripts due to its interactions with $S_{2}$ and that due to its interactions with $\mathrm{P}_{1}\left(m p k^{*}, m s k^{*}\right)$ (a real-world party possessing the same input as the ideal-world party $\left.\mathrm{P}_{1}^{*}\right)$ are P.P.T.-indistinguishable.

At first, consider the transcripts in IA-NMZPoK II $^{\prime} \mathrm{s}$ session. Let IA-NMZPoK $\left.{ }_{\text {II }}{ }^{*}\right)$ and IA-NMZPoK $\left.\mathrm{II}_{\mathrm{II}}\right)$ denote the messages generated by $\mathrm{P}_{1}\left(m p k^{*}, m s k^{*}\right)$ and $S_{2}$ in this session respectively. By IA-NMZPoK II's zero-knowledge property we have

$$
\text { IA-NMZPoK } \left.{ }_{\mathrm{II}}:: \operatorname{Sim}_{2}\left(m p k^{*}, s_{\mathrm{II}}\right) \approx^{\text {PPT }} \text { IA-NM ZPoK }{ }_{\mathrm{II}} *\right)
$$

and by $S_{2}$ 's construction we have

$$
\text { IA-NMZPoK }{ }_{\text {II }}: \operatorname{Sim}_{2}\left(m p k, s_{\text {II }}\right)=\mathrm{IA}_{-\mathrm{NMZPoK}}()
$$


Let $\Omega_{\mathrm{R}}$ denote a random element on group $\mathrm{G}_{2}$. Since $\omega^{*}, \omega, t_{i}{ }^{*}, t_{i}(i=1,2,3,4)$ are probabilistically independent and all are unknown to $A$, from $A$ 's perspectiove we have

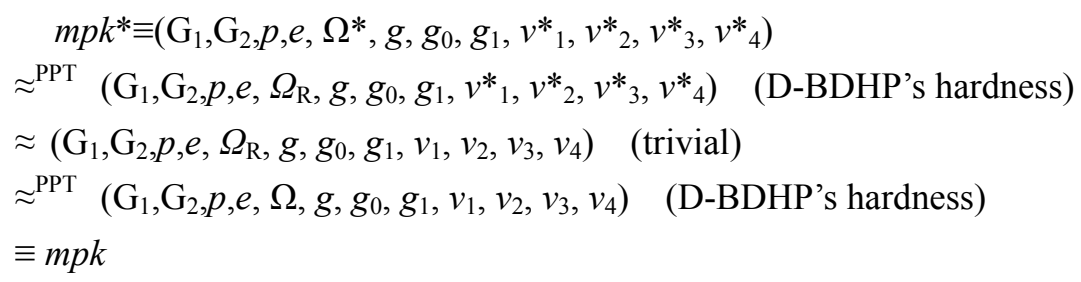

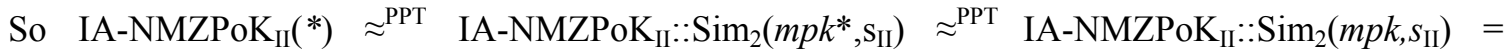
IA-NMZPoK $\mathrm{II}_{\mathrm{I}}$ ).

Now consider the last-round message, which are $d^{*}{ }_{0}\left\|d_{1}{ }^{\prime}|| d_{1}{ }^{\prime \prime}|| d_{2}{ }^{\prime}|| d_{2}{ }^{\prime \prime}\right\| d_{3}{ }^{\prime}\left\|d_{3}{ }^{\prime \prime}\right\| d_{4^{\prime}}{ }^{\prime} \mid d_{4}{ }^{\prime \prime}$ and $d^{*}{ }_{0} \| d^{*_{1}}{ }^{\prime}$ $\left\|d^{*}{ }_{1}{ }^{\prime \prime}|| d^{*}{ }^{\prime}|| d^{*}{ }^{\prime \prime}\right\| d^{*}{ }^{\prime}\left\|d^{*}{ }^{\prime \prime}\right\| d_{4}^{*} \| d^{*} "$ in these two cases( interacting with $S_{2}$ and with $\mathrm{P}_{1}\left(m p k^{*}, m s k^{*}\right)$ ) respectively. Both messages have the same component $d^{*}$, all other components are denoted as $D$ and $D^{*}$ respectively. Expanding $D$ we get

$$
D \equiv d_{1}^{*} / d_{1}^{\prime y_{1}}\left\|d_{1}^{\prime \prime}\right\| d_{2}^{*} / d_{2}^{" y_{2}}\left\|d_{2}^{\prime \prime}\right\| d_{3}^{*} / d_{3}^{" y_{3}}\left\|d_{3}^{"}\right\| d_{4}^{*} / d_{4}^{\prime y_{4}} \| d_{4}^{"}
$$

where $d^{*}{ }_{1}, \quad d_{2}^{*}, \quad d_{3}^{*}, \quad d^{*}{ }_{4}$ come from $\operatorname{UKG}\left(m s k^{*}, a\right)$, i.e., $d^{*}{ }_{1}=g^{-\sigma^{*} t_{2}{ }^{*}}\left(g_{0} g_{1}{ }^{a}\right)^{-\widetilde{r}_{1} t_{2}{ }^{*}}$, $d^{*}{ }_{2}=g^{-\varpi^{*} t_{1} *}\left(g_{0} g_{1}^{a}\right)^{-\widetilde{r}_{1} t^{*}}, d^{*}{ }_{3}=\left(g_{0} g_{1}{ }^{a}\right)^{-\widetilde{r}_{2} t_{4}{ }^{*}}, d^{*}{ }_{4}=\left(g_{0} g_{1}^{a}\right)^{-\widetilde{r}_{2} t_{3}{ }^{*}}$.

\section{Expanding $D^{*}$ we get}

$$
\begin{aligned}
& D^{*} \equiv g^{-\sigma^{*} t_{2}{ }^{*}}\left(h_{1} g_{0}\right)^{-r_{1}^{\prime} t_{2}{ }^{*}} V_{1}^{\sigma t_{2}{ }^{*}}\left\|g^{r_{1}^{\prime} t_{2}{ }^{*}}\right\| g^{-\varpi t_{1}{ }^{*}}\left(h_{2} g_{0}\right)^{-r_{1}^{\prime} t_{1}{ }^{*}} V_{1}^{\sigma t_{1}{ }^{*}}\left\|g^{r_{1}^{\prime} t_{1}{ }^{*}}\right\|\left(h_{3} g_{0}\right)^{-r_{2}^{\prime} t_{4}{ }^{*}} V_{2}^{\sigma t_{4}{ }^{*}} \| \\
& \left\|g^{r_{2}^{\prime} t_{4} *}\right\|\left(h_{4} g_{0}\right)^{-r_{2}^{\prime} t_{3}^{*}} V_{2}^{\sigma t_{3}^{*}} \| g^{r_{2}^{\prime} t_{3} *}
\end{aligned}
$$

where $\sigma, \widetilde{r}_{i}, r_{i}^{\prime}$ and $d_{j}^{\prime \prime}$ are probabilistically independent each other and unkown to $A, \sigma, r_{i}^{\prime}$ are generated by $\mathrm{P}_{1}, d_{j}^{\prime}$ ' by $S_{2}, \widetilde{r}_{i}$ by $F_{\text {Blind-UKG }}^{\text {Boon-Waters }}$.

Since $r_{1}$ ' and $r_{2}$ ' are probabilistically independent each other, $D^{*}$ 's 4 leftmost-components are probabilistically independent of those 4 rightmost-ones; note that $t^{*}{ }_{1}, t^{*}{ }_{2}, t^{*}{ }_{3}, t^{*}{ }_{4}$ are also probabilistically independent each other, we finally partition $D^{*}$ into 4 independent components $D_{\mathrm{i}}^{*}$ as:

$$
\begin{aligned}
& D_{1}{ }^{*} \equiv g^{-\varpi^{*} t_{2}{ }^{*}}\left(h_{1} g_{0}\right)^{-r_{1}^{\prime} t_{2}{ }^{*}} V_{1}^{\sigma t_{2}{ }^{*}}\left\|g^{r_{1} t_{2}{ }^{*}} \quad D_{2}{ }^{*} \equiv g^{-\varpi t_{1}{ }^{*}}\left(h_{2} g_{0}\right)^{-r_{1}^{\prime} t_{1}{ }^{*}} V_{1}^{\sigma t_{1}^{*}}\right\| g^{r_{1}^{\prime} t_{1}{ }^{*}} \\
& D_{3}{ }^{*} \equiv\left(h_{3} g_{0}\right)^{-r_{2}^{\prime} t_{4}{ }^{*}} V_{2}^{\sigma t_{4}{ }^{*}}\left\|g^{r_{2} t_{4} *} \quad D_{4}^{*} \equiv\left(h_{4} g_{0}\right)^{-r_{2}^{\prime} t_{3} *^{*}} V_{2}^{\sigma t_{3}^{*}}\right\| g^{r_{2}^{\prime} t_{3} *}
\end{aligned}
$$

Similarly partition $D$ into 4 independent components $D_{i}$ as:

$$
D_{1} \equiv d_{1}^{*} / d_{1}^{\prime y_{1}}\left\|d_{1}^{\prime \prime} \quad D_{2} \equiv d_{2}^{*} / d_{2}^{n y_{2}}\right\| d_{2}^{\prime \prime} \quad D_{3} \equiv d_{3}^{*} / d_{3}^{\prime \prime y_{3}}\left\|d_{3}^{\prime \prime} \quad D_{4} \equiv d_{4}^{*} / d_{4}^{\prime y_{4}}\right\| d_{4}^{\prime \prime}
$$

The problem is now reduced to analysis on relationship between $D_{i}$ and $D^{*}{ }_{i}$. Consider $D_{3}{ }^{*}$ $\equiv\left(h_{3} g_{0}\right)^{-r_{2}^{\prime} t_{4}^{*}} V_{2}^{\sigma t_{4} *} \| g^{r_{2} t_{4} *}$ and $D_{3} \equiv d_{3}^{*} / d_{3}^{\prime y_{3}} \| d_{3}^{\prime \prime}$ : obviously $D_{3} \approx\left(h_{3} g_{0}\right)^{-\widetilde{r}_{2} t_{4} *} / g^{y_{3} r_{2} t_{4} *} \| g^{r_{2} t_{4} *}$ so it's adequate to analyze the relationship between $\left(h_{3} g_{0}\right)^{-r_{2} t_{4} *^{*}} V_{2}^{\sigma t_{4}{ }^{*}}$ and $\left(g_{0} g_{1}{ }^{a}\right)^{-\widetilde{r}_{2} t_{4}{ }^{*}} / g^{y_{3} r_{2} t_{4} *}$. Further note that $\left(h_{3} g_{0}\right)^{-r_{2}^{\prime} t_{4} *} \approx\left(h_{3} g_{0}\right)^{-\widetilde{r}_{2} t_{4} *}, V_{2}^{\sigma t_{4}{ }^{*}} \approx g^{-y_{3} r_{2} t_{4}{ }^{*}},\left(h_{3} g_{0}\right)^{-\widetilde{r}_{2} t_{4} *}$ and $g^{r_{2} t_{4}{ }^{*}}$ are independent each other, so $D_{3} * \approx D_{3}$. For the same reason $D_{4} * \approx D_{4}$.

Consider $D_{1}{ }^{*} \equiv g^{-\varpi^{*} t_{2}{ }^{*}}\left(h_{1} g_{0}\right)^{-r_{1}^{\prime} t_{2}{ }^{*}} V_{1}^{\sigma t_{2}{ }^{*}} \| g^{r_{1} t_{2}{ }^{*}}$ and $D_{1} \equiv d_{1}^{*} / d_{1}^{\prime y_{1}} \| d_{1}^{\prime \prime}$ : obviously $D_{1} \approx g^{-\omega^{*} t_{2}{ }^{*}}\left(g_{0} g_{1}{ }^{a}\right)^{-\widetilde{r}_{1} t_{2}{ }^{*}} / g^{r_{1} t_{2}{ }^{*} y_{1}} \| g^{r_{1} t_{2}{ }^{*}}$, by similar analysis as before we have $D_{1} * \approx D_{1}$. For the same reason $D_{2} * \approx D_{2}$. Therefore:

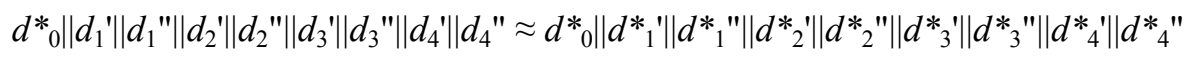

In consequence, under the assumption of D-BDHP's hardness on $\mathrm{J}$, from $A$ 's perspective the transcripts due to its interactions with $S_{2}$ and that due to its interactions with $\mathrm{P}_{1}\left(m p k^{*}, m s k^{*}\right)$ are P.P.T.-indistinguishable. In particular, $A$ 's output in the former case is P.P.T.-indistinguishable from its 
output in the latter, the error is (by some straightforward calculation) upper-bounded by $\eta_{\mathrm{III}}$ $+2 A d v_{J}^{D-B D H P}(k)$ where $\eta_{\mathrm{III}}$ is IA-NMZPoK ${ }_{\mathrm{III}}$ 's knowledge extractor's error function. As a result,

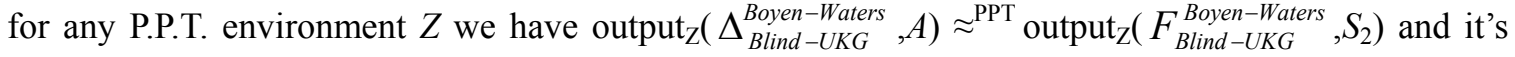
easy to estimate $S_{2}$ 's time-complexity $\mathrm{T}_{S 2}=\mathrm{T}_{A}+\mathrm{T}_{e I I I}+\mathrm{O}(1)$ where $\mathrm{T}_{A}$ and $\mathrm{T}_{\text {eIII }}$ are $A$ 's and IA-NMZPoK ${ }_{\text {III }}$ 's extractor's computation-time.

Combining all consequences in the above, the theorem is finally proved.

\section{APPENDIX.D IA-NMZPoK PROTOCOL'S CONSTRUCTION}

\section{D.1 (Dense) $\Omega$-Protocol ${ }^{[8,14]}$}

A $\Omega$-protocol for a given relation $R$ is a 3-move protocol in CRS model consisted of P.P.T. algorithms $\mathrm{D}, \mathrm{A}, \mathrm{Z}, \Phi, \mathrm{Sim}$ and $\mathrm{Ext}=\left(\mathrm{Ext}_{1}, \mathrm{Ext}_{2}\right)$. D is the CRS generating algorithm. All algorithms except D takes a CRS $\omega$ as one of its inputs. For some $(x, w)$ s.t. $R(x, w)=1$ the common input for both the prover $\mathrm{P}$ and the verifier $\mathrm{V}$ is $x$ and witness $w$ is P's private input. In the first move $\mathrm{P}$ generates a randomness $r$, computes $a \leftarrow \mathrm{A}(\omega, x, w, r)$ and sends $a$ to $\mathrm{V}$; in the second move, $\mathrm{V}$ selects a challenge $c$ at random and sends it back to $\mathrm{P}$; then $\mathrm{P}$ computes $\mathrm{Z} \leftarrow \mathrm{Z}(\omega, x, w, r, c)$ and sends $z$ to $\mathrm{V}$ in the last move; on receiving $z, \mathrm{~V}$ outputs "accept" or "refuse" depending on whether $\Phi(\omega, x, a, c, \mathrm{z})=1$ or 0 . In addition, a $\Omega$-protocol has the following properties [14]:

(1) For the honest $\mathrm{P}$ which behaves under the above specification, $\Phi(\omega, x, a, c, z)=1$ is always true.

(2) Given $c$ and $x \in L_{\mathrm{R}}$ the simulator $\operatorname{Sim}(\omega, x, c)$ can generate accepting transcripts with a distribution that is P.P.T.-indistinguishable from those when $\mathrm{P}$ and $\mathrm{V}$ execute the protocol on common input $x$ while $\mathrm{V}$ selects $c$ as the challenge.

(3) $(\sigma, \tau) \leftarrow \operatorname{Ext}_{1}(k)$ where $\sigma$ is P.P.T.-indistinguishable from $\omega \leftarrow \mathrm{D}(k)$; in addition, if there exists two accepting transcripts $(a, c, z)$ and $\left(a, c^{\prime}, z^{\prime}\right)$ where $c \neq c^{\prime}$ for some given $x \in L_{\mathrm{R}}$, then $\operatorname{Ext}_{2}(x, \tau,(a, c, z))$ outputs $w$ such that $\mathrm{R}(x, w)=1$.

A dense $\Omega$-protocol has the additional property as follows [6]:

(4) The CRS-domain D is a subset of a larger domain, D*(named extended CRS-domain), which is an Abelian group and its group operations are all efficient. Furthermore, the element of $\mathrm{D}$ and $\mathrm{D}^{*}$ is P.P.T.-indistinguishable from each other.

\section{D.2 A General Construction for IA-NMZPoK Protocol}

Now we present a general construction for IA-NMZPoK protocol(definition 2.3-2.4) for given relation $R$. It uses a secure (strong existential-unforgeable) one-time signature scheme, a secure IBTC sheme(definition 2.5) and a dense $\Omega$-protocol as its components. Note that among these components the secure one-time signature scheme and IBTC scheme can all be efficiently constructed, only the $\Omega$-protocol is related with the specific relation $R$, therefore the construction can be regarded as a general transformation from the (comparatively weak but easy to construct) $\Omega$-protocol to the 


\section{IA-NMZPoK protocol.}

This construction is similar as that in [14] and borrows the coin-tossing technique used in [5-6]. Given a binary relation $\mathrm{R}$ and its dense $\Omega$-protocol $\Omega_{\mathrm{R}}=\left(\mathrm{D}, \mathrm{A}, \mathrm{Z}, \Phi, \mathrm{Sim}, \mathrm{Ext}=\left(\mathrm{Ext}_{1}, \mathrm{Ext}_{2}\right)\right)$ with its $\mathrm{CRS}$ denoted as $\omega ; \mathrm{SIG}=(\mathrm{KGen}, \mathrm{Sign}, \mathrm{Vf})$ is a strong existential-unforgeable one-time signature scheme; IBTC $=\left(D_{\mathrm{TC}}\right.$, Setup,UKG,Cmt,Vf,FakeCmt, FakeDmt $)$ is a secure IBTC scheme with its master public/secret-key pair denoted as $\left(m p k_{\mathrm{TC}}, m s k_{\mathrm{TC}}\right)$. The constructed protocol IA-NMZPoK $\mathrm{R}_{\mathrm{R}}$ (see Figure D.1) is in the ACRS model and its ACRS is the IBTC scheme's master public-key $m p k_{\mathrm{TC}}$.

For clearity, we use IBTC::Cmt to stand for IBTC scheme's commitment algorithm Cmt, SIG::Sign to stand for SIG scheme's signing algorithm Sign, etc. $P$ and $V$ denote the prover(P)'s and verifier(V)'s identities respectively. $\xi$ denotes the protocol's transcripts excluding the signature, i.e., $\xi \equiv k_{1}\left\|\omega_{2}\right\| \omega_{1}\left\|d_{1}\right\|$ sig_ $v k\|c m t\| c \||||d m t| \mid z$. Actually the first 3-move session is an IBTC-based coin-tossing [5-6] to generate a CRS $\omega$ for the following protocol $\Omega_{R}$ and the second 3-move session is similar as the construction of NMZPoK protocol in [14].

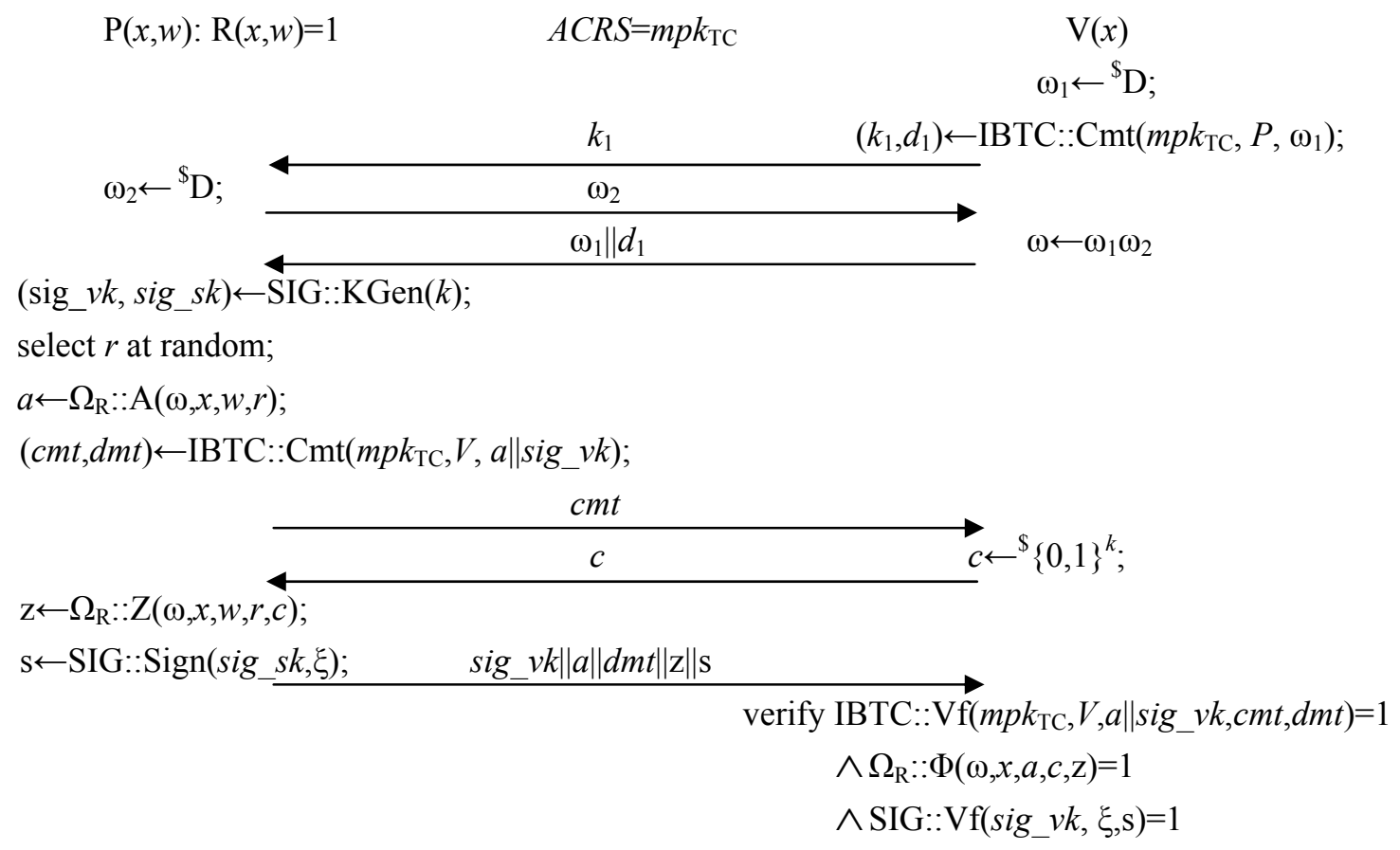

Figure D.1 IA-NMZPoK protocol IA-NMZPoK ${ }_{R}$ for relation R.

Theorem D.1 IA-NMZPoK $\mathrm{K}_{\mathrm{R}}$ is an IA-NMZPoK protocol for relation R.

Proof sketch The proof is similar as that of [14]'s theorem 4.1-4.2, the most difference is the simulation algorithm $\operatorname{Sim}=\left(\operatorname{Sim}_{1}, \operatorname{Sim}_{2}\right)$ and the extraction algorithm $\operatorname{Ext}=\left(\operatorname{Ext}_{1}, \mathrm{Ext}_{2}\right)$ which are presented here.

Let $\quad u s k(P) \equiv \mathrm{IBTC}:: \mathrm{UKG}\left(m s k_{\mathrm{TC}}, P\right), \quad u s k(V) \equiv \mathrm{IBTC}:: \mathrm{UKG}\left(m s k_{\mathrm{TC}}, V\right) . \quad \operatorname{Sim}_{1}(u s k(V)) \quad$ normally simulates the coin-tossing (the first 3-move session in IA-NMZPoK $\mathrm{K}_{\mathrm{R}}$ ) as specified in the constrcution and its simulated transcript is denoted as $k_{1}\left\|\omega_{2}\right\| \omega_{1} \| d_{1}$, then it outputs $k_{1}\left\|\omega_{2}\right\| \omega_{1}\left\|d_{1}\right\| u s k(V)$. $\operatorname{Sim}_{2}\left(m p k_{\mathrm{TC}}, x, \omega, k_{1}\left\|\omega_{2}\right\| \omega_{1}\left\|d_{1}\right\| u s k(V)\right)\left(\right.$ where $\left.\omega=\omega_{1} \omega_{2}\right)$ computes $(\overline{c m t}, \lambda) \leftarrow \operatorname{IBTC}::$ FakeCmt $\left(m p k_{\mathrm{TC}}\right.$, 
$V$, usk $(V))$ and (sig_vk,sig_sk) $\leftarrow \operatorname{SIG}:: \operatorname{KGen}(k)$, selects $c$ at random, computes $(a, \mathrm{z}) \leftarrow \Omega_{\mathrm{R}}:: \operatorname{Sim}(\omega, x, c)$, $\bar{d} \leftarrow$ FakeDmt $\left(m p k_{\mathrm{TC}}, a \| s i g \_v k, \lambda, \overline{c m t}\right), \mathrm{s} \leftarrow \operatorname{SIG}:: \operatorname{Sign}\left(s i g \_s k, \xi\right)$ where $\xi$ is the whole transcript (as specified in the construction) excluding the signature s. Finally $\operatorname{Sim}_{2}$ outputs

$$
k_{1}\left\|\omega_{2}\right\| \omega_{1}\left\|d_{1}\right\| c m t\|c\| s i g \_v k\|a\| \bar{d}\|z\| s
$$

For the extractor $\operatorname{Ext}=\left(\operatorname{Ext}_{1}, \operatorname{Ext}_{2}\right), \operatorname{Ext}_{1}(u s k(P))$ computes $(\omega, \tau) \leftarrow \Omega_{\mathrm{R}}:: \operatorname{Ext}_{1}(k)$ and outputs $(\omega$, $u s k(P) \| \tau)$. $\operatorname{Ext}_{2}\left(m p k_{\mathrm{TC}}, \omega, u s k(P) \| \tau\right)$ computes $\left(\overline{k_{1}}, \lambda_{1}\right) \leftarrow \operatorname{IBTC}::$ FakeCmt $\left(m p k_{\mathrm{TC}}, P\right.$, usk $\left.(P)\right)$ and sends $\overline{k_{1}}$ out; on receiving $\omega_{2}$, it computes $\omega_{1} \leftarrow \omega / \omega_{2}, \overline{d_{1}} \leftarrow$ FakeDmt $\left(m p k_{\mathrm{TC}}, \omega_{1}, \lambda_{1}, \overline{k_{1}}\right)$ and responses with $\omega_{1} \| \overline{d_{1}}$; then it randomly generates a challenge $c$ on receiving $c m t$. When it gets the last message sig_vk\|a\|dmt||z||s, it checks all the required conditions and call $\Omega_{\mathrm{R}}:: \operatorname{Ext}_{2}(\omega, x, \tau, a \| c|| z)$.

Now it can be shown that $\operatorname{Sim}=\left(\operatorname{Sim}_{1}, \operatorname{Sim}_{2}\right)$ and $\operatorname{Ext}=\left(\operatorname{Ext}_{1}, \operatorname{Ext}_{2}\right)$ indeed satisfy the properties in definition 2.3-2.4, the analysis is almost the same as in the proof of [14]'s theorem 4.1-4.2. 